\title{
Sobre a radiação cósmica de fundo de micro-onda ${ }^{+*}$
}

José Maria Filardo Bassalo ${ }^{1}$

Academia Paraense de Ciências

Belém - PA

M. Cattani ${ }^{2}$

Instituto de Física - USP

São Paulo - SP

\section{Resumo}

Neste artigo, procuraremos dar uma pálida ideia para o leitor do que poderia ser a Radiação Cósmica de Fundo de Micro-onda (RCFM) que, segundo o tradicional modelo do "Big Bang", foi gerada por uma explosão primordial. Com esse objetivo, achamos muito importante apresentarmos um breve resumo histórico de como o Microcosmo, baseado no Modelo Padrão da Física das Partículas Elementares (MPPE), e o Macrocosmo, baseado no Modelo Padrão do Big Bang (MPBB), evoluíram no tempo. Além disso, na parte final do artigo analisaremos os dois processos físicos apresentados na literatura que procuram explicar a RCFM: Bariogênese e Plasma Quark-Glúon.

Palavras-chave: Radiação Cósmica de Fundo de Micro-onda; Modelo Padrão da Física das Partículas Elementares; Modelo Padrão do Big Bang; Bariogênese; Plasma Quark-Glúon.

\footnotetext{
Abstract

${ }^{+}$On the Cosmic Microwave Background Radiation

* Recebido: julho de 2017.

Aceito: setembro de 2017.

${ }^{1}$ E-mail: jmfbassalo@gmail.com, ${ }^{2}$ E-mail: mcattani@if.usp.br
}

In this article we will try to give a pale idea to the reader of what could be the Cosmic Microwave Background (RCFM) that, according to the traditional Big Bang model, was generated by a primordial explosion. With this purpose we find it very important to present a brief historical 
summary of how the Microcosm, based on the Standard Model of Elementary Particle Physics (MPPE), and the Macrocosm, based on the Standard Big Bang Model (MPBB), have evolved over time. In addition, in the final part of the article we will analyze the two physical processes presented in the literature that seek to explain the RCFM: Bariogenesis and Plasma Quark-Gluon.

Keywords: Cosmic Microwave Background Radiation; Standard Model of Elementary Particle Physics; Standard Big Bang Model; Bariogenesis; Plasma Quark-Gluon.

\section{Introdução}

Para entendermos a Radiação Cósmica de Fundo de Micro-onda (RCFM) é necessário fazermos um resumo histórico (obedecendo a uma ordem cronológica) do micromundo, estudado pelo Modelo Padrão da Física das Partículas Elementares (MPPE) e do macromundo, traduzido pelo Modelo Padrão do Big Bang (MPBB). Para esses resumos, usaremos os textos: Steven Weinberg, Os Três Primeiros Minutos: Uma Discussão Moderna Sobre a Origem do Universo²; Sheldon Lee Glashow and Ben(jamin) William Bova, Interactions: A Journey Through the Mind of a Particle Physicist and the Matter of This World ${ }^{3}$; Leon Max Lederman and Dick Teresi, The God Particle ${ }^{4}$; Abraham Pais, Inward Bound of Matter and Forces in the Physical World5; John Gribbin, Q is for Quantum: An Encyclopaedia of Particle Physics6; Richard Morris, O Que Sabemos Sobre o Universo: Realidade e Imaginação Científica $^{7}$; Maria Cristina Batoni Abdalla, O Discreto Charme das Partículas Elementares ${ }^{8}$; Neil de Grasse Tyson e Donald Goldsmith, Origens: Catorze Bilhões de Anos de Evolução Cósmica9; e Neil de Grasse Tyson, Morte no Buraco Negro e Outros Dilemas Cósmicos ${ }^{10}$.

\footnotetext{
2 Guanabara Dois, 1980.

${ }^{3}$ Warner Books, 1989.

${ }^{4}$ Delta Book, 1994.

5 Clarendon Press and Oxford University Press, 1995.

6 Touchstone Book, 1999.

7 Jorge Zahar Editor, 2001.

${ }^{8}$ Editora da Universidade do Estado de São Paulo, 2006; Livraria da Física, 2016.

${ }^{9}$ Planeta do Brasil Ltda., 2016.

10 Planeta do Brasil Ltda., 2016.
} 


\section{Modelo Padrão da Física das Partículas Elementares}

Iniciemos com um pequeno resumo cronológico do MPPE. Em 189511, o físico alemão Wilhelm Conrad Roentgen (1845-1923; PNF, 1901) descobriu os raios-X. Logo a seguir, em 189612, o físico francês Antoine Henry Becquerel (1852-1908; PNF, 1903) encontrou que havia uma relação entre a luminescência (fluorescência e fosforescência) e os raios-X, pois observou que cristais de sulfato de urânio-potássio [contendo uranilo: óxido de urânio $\left(\mathrm{UO}_{2}\right)$ ] eram capazes de impressionar uma chapa fotográfica recoberta com papel escuro, estando o conjunto exposto à luz solar. Esses "raios" emitidos pelo uranilo ficaram inicialmente conhecidos como "raios (de) Becquerel". Note-se que a descoberta dos raios-X levou o físico e químico inglês-neozelandês Sir Ernest Rutherford (1871-1937; PNQ, 1908) a medir, em $1896^{13}$, a ionização provocada por esses "raios".

Em 1896, o físico holandês Peter Zeeman (1865-1943; PNF, 1902) começou a estudar a influência do campo magnético $(\mathrm{H}$, de módulo $\mathrm{H})$ sobre o estado de polarização da luz, que havia sido objeto de pesquisa do físico e químico inglês Michael Faraday (1791-1867), o famoso efeito Faraday (publicado em 1846) ${ }^{14}$. Desse modo, trabalhando com um equipamento melhor do que o utilizado por Faraday, ou seja, uma bobina de Rühmkorff [inventada, em 1851, pelo mecânico e eletricista alemão Heinrich Daniel Rühmkorff (1803-1877)], que produzia um $\mathrm{H}$ da ordem de 10 kilogauss, e uma grade de difração [que o físico norte-americano Henry August Rowland (1848-1901) construíra, em 188215, com um raio de 10 pés, possuindo 14.938 linhas por polegada, e apresentando um poder de resolução acima de 150.000], Zeeman observou, naquele ano de 1896, que as duas linhas amarelas D do sódio (Na) eram alargadas quando examinadas sob a ação de um $\mathrm{H}$ muito forte. Ele ainda observou que tais linhas eram circularmente polarizadas quando observadas paralelamente às linhas de força do campo magnético, e linearmente plano-polarizadas quando a observação era dirigida perpendicularmente a essas mesmas linhas de força. Alargamentos semelhantes ao do espectro do Na foram ainda observados por Zeeman com outras linhas espectrais. Essa observação (depois conhecida como efeito Zeeman) foi publicada, ainda em $1896^{16}$.

\footnotetext{
${ }^{11}$ Sitzungsberichte der Würzburger Physikalischen-Medicinischen Gesellschaft, v. 137, p. 132, 1895.

12 Comptes Rendus Hebdomadaires des Séances de l’Académie des Sciences de Paris (CRHSASP), v. 122, p. 420; 501, 1896.

${ }^{13}$ Philosophical Magazine, v. 42, p. 392, 1896.

${ }^{14}$ Philosophical Transactions of the Royal Society of London, p. 1; Philosophical Magazine, v. 28, p. 345, 1846.

15 Philosophical Magazine, v. 13, p. 469; Nature, v. 26, p. 211, 1882.

16 Verlag van de Gewone Vergadering der wis-en Natuurkindige Afdeeling, Koniklijke Akademie van Wetenschappen te Amsterdam, v. 5, p. 181; 242; Verbandlungen der Physikalischen Gesellschaft zu Berlin, v. 7, p. 128, 1896.
} 
Logo depois, em 189717, o físico holandês Hendrik Antoon Lorentz (1853-1928; PNF, 1902) deu uma explicação teórica para esse novo fenômeno observado por Zeeman. Vejamos como: em 189218, ele desenvolveu sua famosa Teoria do Elétron, segundo a qual a eletricidade possuía uma estrutura composta de "partículas carregadas" (que as denominou de íons, em 1895), e considerou que as mesmas estavam presas aos átomos por uma força do tipo elástica e sujeitos à ação de uma força externa e, então, demonstrou que, na presença de um campo magnético $\mathrm{H}(\mathrm{H})$, esses íons oscilavam na direção desse campo com a frequência própria $v_{0}$, enquanto giravam em órbitas circulares em planos normais à direção de $\mathrm{H}(\mathrm{H})$ com a frequência $v$ dada e traduzida pela expressão (na notação atual): $v=v_{0} \pm e H /\left(4 \pi m_{e} c\right)$, onde $e$ e $m_{e}$ representam, respectivamente, a carga e a massa do íon, e $c$ é a velocidade da luz no vácuo. Ainda segundo Lorentz, quando a observação do efeito Zeeman era feita na direção do campo magnético, apareciam apenas duas linhas polarizadas circularmente e em sentido inverso uma da outra. Por outro lado, quando a observação era feita perpendicularmente ao campo $\mathrm{H}$, apareciam três linhas, sendo a central polarizada linearmente à direção de $\mathrm{H}$, a chamada componente $\pi$, e as duas extremas, polarizadas também linearmente, porém perpendicularmente à direção de $\mathrm{H}$, a chamada componente $\sigma$ (essa denominação deriva da palavra alemã senkrecht que significa perpendicular). Essa previsão de Lorentz [desdobramento (spliting) de linhas espectrais] foi confirmada por Zeeman, pela primeira vez e ainda em 189719, observando a linha azul $(4800 \AA$ ) do cádmio $(\mathrm{Cd})$ sob a ação de um campo magnético de 32 kilogauss. O desdobramento de linhas espectrais sob a ação de um campo magnético forte foi também observado, ainda em 1897, em outros laboratórios do mundo pelos físicos: o norteamericano Albert Abraham Michelson (1852-1931; PNF, 1907)20; o francês Marie-Alfred Cornu (1841-1902)21; e o alemão C. G. Walther König22.

É interessante registrar que, em 189723, o físico inglês Sir Joseph John Thomson (1856-1940; PNF, 1906) descobriu que os raios catódicos [descobertos pelo físico alemão Eugen Goldstein (1850-1931), em 187624] constituíam a corrente elétrica, ou seja, que esta era composta de "cargas elétricas negativas" (os íons de Lorentz), ocasião em que calculou a relação entre a carga elétrica (e) e a massa $\left(\mathrm{m}_{\mathrm{e}}\right)$ das mesmas $\left(\mathrm{e} / \mathrm{m}_{\mathrm{e}}\right)$. Quando Lorentz as denominou de elétrons, em 1899, Thomson passou a ser considerado o descobridor do elétron $\left(\mathrm{e}^{-}\right)$.

\footnotetext{
17 Annalen der Physik, v. 63, p. 278; Philosophical Magazine, v. 43, p. 232, 1897.

18 Archives Néerlandaises des Sciences Exactes et Naturales, v. 25, p. 365, 1892.

19 Verlag van de Gewone Vergadering der wis-en Natuurkindige Afdeeling, Koniklijke Akademie van Wetenschappen te Amsterdam, v. 6, p. 13; 99; 260, 1897.

20 Philosophical Magazine, v. 44, p. 109, 1897.

21 Comptes Rendus de l'Académie des Sciences de Paris, v. 125, p. 555, 1897.

22 Annalen der Physik, v. 62, p. 240, 1897.

23 Philosophical Magazine, v. 44, p. 295, 1897.

${ }^{24}$ Monatsberichte der Königlich Preussischen Akademie de Wissenschaften zu Berlin, p. 279, 1876.
} 
Destaca-se que, também em 189725, o físico inglês Sir Joseph J. Larmor (1857-1942) apresentou outra explicação teórica para o efeito Zeeman. Segundo ele, o efeito magnético de um campo magnético sobre partículas carregadas que descrevem órbitas circulares era o de superpor à frequência própria de rotação uma frequência precessional em torno de $\mathrm{H}$ e de mesmo valor calculado por Lorentz. Essa frequência precessional ficou conhecida como a frequência de Larmor $\left(v_{\mathrm{L}}\right)$ e dada pela seguinte expressão (em notação atual): $v_{\mathrm{L}}=\mathrm{e} /\left(2 \mathrm{~m}_{\mathrm{e}}\right) \times$ $\mathrm{H} /(2 \pi \mathrm{c})$. Destaque-se que foi também nesse artigo que Larmor demonstrou que uma carga elétrica acelerada irradia energia, depois conhecida como radiação de Larmor.

$\mathrm{O}$ estudo do efeito Zeeman continuou despertando muito interesse no mundo inteiro. Contudo, novas observações experimentais sob a ação de um campo magnético em uma linha espectral não se ajustaram na explicação de Lorentz-Larmor. Com efeito, logo em $1898^{26}$, o físico irlandês Thomas Preston (1860-1900) observou que as linhas azuis do Zn (4722A) e do

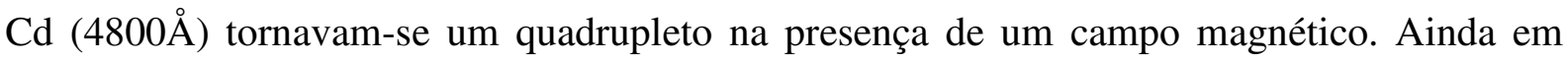
$1898^{27}$, Cornu obteve um outro quadrupleto, desta vez para a linha $\mathrm{D}_{1}$ do $\mathrm{Na}$ e um sextupleto para a linha $\mathrm{D}_{2}$, também do Na, resultado esse que foi logo confirmado por Preston. Também

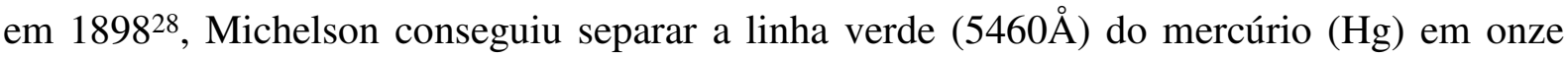
componentes. Além disso, e no mesmo ano de $1898^{29}$, quadrupletos e outras estruturas complexas foram obtidas pelos físicos franceses Becquerel e Henri Alexandre Deslandres (18531948), examinando linhas espectrais do ferro $(\mathrm{Fe})$, no espectro visível e no ultravioleta.

O "efeito Zeeman complexo" (nome cunhado por Lorentz ao conjunto das linhas espectrais magnéticas relacionadas acima) foi estudado sistematicamente por Preston e, em consequência desses estudos, ele formulou, em 189930, uma lei denominada de Lei Geral do Fenômeno da Perturbação Magnética das Linhas Espectrais, segundo a qual, as séries espectrais de uma dada substância apresentam o mesmo padrão (pattern) de componentes na presença de um campo magnético; por outro lado, linhas espectrais de diferentes elementos da mesma família (por exemplo, os álcalis ou as terras raras), têm o mesmo efeito Zeeman. Lorenz tentou, sem sucesso, explicar essa regra de Preston (RP), ainda em 189931, usando sua Teoria do Elétron. Também em 1899, o físico alemão Woldemar Voigt (1850-1919) 32 e, independentemente, D. A. Goldhammer (1860-1922) ${ }^{33}$ estudaram o chamado efeito Zeeman

\footnotetext{
25 Philosophical Magazine, v. 44, p. 503, 1897.

26 Scientific Transactions of the Royal Dublin Society, v. 6, p. 385, 1898.

27 Comptes Rendus de l'Académie des Sciences de Paris, v. 126, p. 181, 1898.

28 Astrophysical Journal, v. 7, p. 131, 1898.

${ }^{29}$ Comptes Rendus de l'Académie des Sciences de Paris, v. 126, p. 997; 127, p. 18, 1898.

${ }^{30}$ Nature, v. 59, p. 248, 1899.

31 Physikalische Zeitschrift, v. 1, p. 39, 1899.

32 Annalen der Physik, v. 67, p. 345, 1899.

33 Annalen der Physik, v. 67, p. 696, 1899.
} 
inverso, ou seja, eles analisaram o que acontecia com o espectro de uma chama de Na colocada em um campo magnético, quando essa chama é atravessada por uma luz plano-polarizada de frequência próxima à da linha $\mathrm{D}$ daquele elemento químico.

Em fins de 1897, a física e química polonesa Marie Sklodowska Curie (1867-1934; PNF, 1903; PNQ, 1911) (Madame Curie) folheou os CRHSASP em busca de um assunto para a sua Tese de Doutoramento e deteve-se diante dos trabalhos de Becquerel. Imediatamente passou a estudar os "raios de Becquerel", expressão usada inicialmente por ela própria. Para tal estudo, utilizou a piezoeletricidade, que havia sido descoberta por seu marido, o físico francês Pierre Curie (1859-1906; PNF, 1903) (eles se casaram em 1895) juntamente com seu irmão, o químico francês Paul-Jacques Curie (1855-1941), em 188034. Os "raios de Becquerel" ionizavam o ar e o tornava capaz de conduzir corrente elétrica. Essa corrente era detectada por um galvanômetro, podendo, no entanto, ser neutralizada por intermédio de um potencial piezoelétrico gerado pela pressão de um cristal. O valor dessa pressão media a intensidade dos "raios de Becquerel". Ao estudar o tório (Th), em 1898, Madame Curie observou que esse elemento químico se comportava como o uranilo de Becquerel. Foi por essa ocasião que Madame Curie denominou radioatividade esse novo fenômeno físico. No prosseguimento de suas pesquisas sobre esse novo fenômeno físico (agora auxiliada por seu marido Pierre Curie), passou a estudar a "pechblenda" $\left(\mathrm{UO}_{2}\right)$. Com esse estudo, o casal Curie conseguiu isolar um novo elemento químico, vizinho do bismuto (Bi), ao qual chamou de polônio (Po), em homenagem à pátria de Madame Curie. Tal descoberta foi anunciada nos CRHSASP 127, p. 1001, de julho de 1898. No CRHSASP 127, p. 1215, de dezembro de 1898, o casal Curie, com a colaboração do químico francês Gustave Bémont (1857-1932), anunciou a existência de outro elemento radioativo: o rádio $(\mathrm{Ra})$.

Também em 189835, Rutherford mediu a ionização provocada pelos "raios de Becquerel" fazendo-os passar através de folhas metálicas. Neste seu trabalho, ele descobriu que os mesmos eram constituídos de dois tipos de decaimento ("decay"): decaimento (raios/partículas) alfa ( $\alpha$-decay), carregada positivamente, e decaimento (raios/partículas) beta ( $\beta$-decay), carregada negativamente. Em 1900, vários outros resultados foram encontrados para aumentar o conhecimento da radioatividade. Com efeito: 1) Becquerel mostrou que os raios $\beta$ eram elétrons ${ }^{36} ; 2$ ) o físico francês Paul Villard (1860-1934) ${ }^{37}$ observou que a radioatividade possuía uma terceira parcela que não era defletida pelo campo magnético, parcela essa penetrante e semelhante aos raios- $X$, à qual Rutherford denominou de decaimento (raios) gama $(\gamma$-decay), constituída de radiação pura; note-se que esta descoberta foi confirmada por

\footnotetext{
34 CRHSASP, v. 91, p. 294, 1880.

35 Proceedings of the Cambridge Philosophical Society, v. 9, p. 401, 1898.

36 CRHSASP, v. 130, p. 809, 1900.

37 CRHSASP, v. 130, p. 1010; 1178, 1900.
} 
Becquerel $^{38}$; e 3) Rutherford ${ }^{39}$ percebeu que havia certa irregularidade no produto gasoso radioativo que escapava do tório (Th). Por haver incerteza na natureza desse produto radioativo, Rutherford deu a esse "novo" gás o nome "emanação". Registre-se que foi nesse trabalho que ele introduziu o conceito de meia-vida $\left(\mathrm{T}_{1 / 2}\right)$ - o tempo que uma amostra radioativa leva para reduzir-se à metade da amostra.

Até o final de 1900, a Física era denominada Clássica, cuja característica fundamental era a energia contínua. Em dezembro de $1900^{40}$, o físico alemão Max Karl Ernest Planck (1858-1947; PNF, 1918) propôs que a energia $(\varepsilon)$ dos osciladores harmônicos de frequência (v), variava discretamente e formada do quantum de energia: $\varepsilon=\mathrm{h} v$, sendo h a hoje conhecida constante de Planck. Iniciava-se, desse modo, a era da energia discreta (quântica) da Física, que domina hoje o MPPE.

Com o propósito de testar a RP, os físicos alemães Carl David Tomé Runge (18561927) e Louis Carl Heinrich Friedrich Paschen (1865-1947), em 190041, investigaram algumas linhas do $\mathrm{Hg}$ e observaram que, sob a ação de um campo magnético, a linha verde

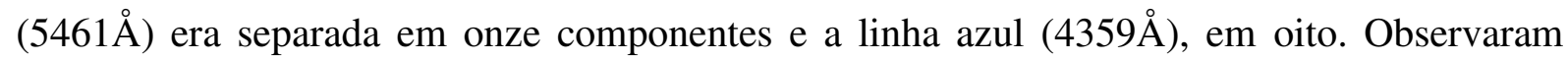

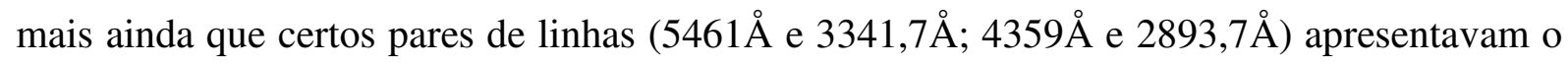
mesmo número de "componentes Zeeman", igualmente separados em frequência. Mais tarde, em 190242, analisando as séries de tripletos do magnésio $(\mathrm{Mg}), \mathrm{Cd}$ e $\mathrm{Hg}$, e as séries de dupletos do cobre $(\mathrm{Cu})$, prata $(\mathrm{Ag})$, alumínio $(\mathrm{A} \ell)$, tálio $(\mathrm{T} \ell)$ e $\mathrm{Na}$ (linhas $\mathrm{D})$, Runge observou que em todos os casos das linhas correspondentes, elas apresentavam o mesmo número de componentes magnéticos com a mesma diferença de frequências.

Ainda em 190243, Rutherford confirmou que os raios $\gamma$ não eram desviados por campo magnéticos e, em vista disso, afirmou que eles eram raios $\beta$ "duros". Também em 190244, mas agora ensinando e pesquisando na Universidade de McGill, em Montreal, Canadá, Rutherford e o químico inglês Frederick Soddy (1877-1956; PNQ, 1921), formularam a teoria de que cada processo radioativo é uma "transmutação de elementos". Em 190645, Rutherford realizou a primeira experiência (hoje conhecida como reação nuclear), na qual estudou o espalhamento de partículas $\alpha$ através de uma lâmina de mica de $0,003 \mathrm{~cm}$ de espessura.

\footnotetext{
38 CRHSASP, v. 130, p. 1154, 1900.

39 Philosophical Magazine, v. 49, p. 1, 1900.

${ }^{40}$ Verhandlungen der Deutschen Physikalische Gesellschaft, v. 2, p. 237, 1900.

${ }^{41}$ Physikalische Zeitschrift, v. 1, p. 480, 1900.

42 Physikalische Zeitschrift, v. 3, p. 441, 1902.

${ }^{43}$ Physikalische Zeitschrift, v. 3, p. 517, 1902.

${ }^{44}$ Philosophical Magazine, v. 4, p. 370; 569, 1902.

45 Philosophical Magazine, v. 11, p. 166; 12, p. 134, 1906.
} 
O "efeito Zeeman complexo" foi analisado por Runge em 190746 com o objetivo de ajustar numericamente as diferenças de frequências $(\Delta v)$ entre os componentes magnéticos. Neste trabalho, Runge encontrou que as distâncias (medidas em $\Delta v$ ) desses componentes ao componente central, são múltiplas da quantidade $v_{\mathrm{L}} / \mathrm{r}$, onde $\mathrm{r}$ é um número inteiro compreendido entre 1 ["tripleto normal Zeeman" - conhecido posteriormente como efeito Zeeman normal (EZN)] e 12, conhecido como "número de Runge”. Note-se que Runge deduziu esse resultado, que posteriormente veio a ser conhecido como Regra de Runge (RR), depois de cuidadosa análise matemática dos dados experimentais até então conhecidos.

Logo após Runge haver anunciado a sua regra, um resultado experimental obtido por W. Lohmann, em 190847, começou a colocar em dúvida as RR e RP. Em seu trabalho, Lohmann mostrou que a grande maioria das linhas do hélio (He) (com as quais trabalhou em sua Tese de Doutorado defendida em 1907 na Universidade de Halle) era separada apenas em “tripleto normal Zeeman", independentemente das séries às quais aquelas linhas pertenciam.

Também em $1908^{48}$, Rutherford e o físico alemão Hans (Johannes) Wilhelm Geiger (1882-1945), usando métodos espectroscópicos, descobriram que as $\alpha$ eram átomos de hélio $\left({ }_{2} \mathrm{He}^{4}\right)$ duplamente ionizados. Ainda em $1908^{49}$, Geiger estudou o espalhamento de um feixe de $\alpha$, oriundo de um composto de rádio, o brometo de rádio $\left(\mathrm{RaBr}_{2}\right)$, através de uma lâmina fina de metal [alumínio $(\mathrm{A} \ell)$ e ouro $(\mathrm{Au})$ ]. As $\alpha$ espalhadas eram detectadas em contadores de cintilações. Usando essa técnica de contagem, Geiger e o físico inglês Ernst Marsden (18891970), em 190950, estudaram o espalhamento de um feixe de $\alpha$ [oriundas do radônio (Rn)], através de uma lâmina fina de metal. Nesse estudo, eles observaram que do feixe, não muito bem colimado e contendo cerca de 8.000 daquelas partículas, apenas uma delas era refletida, ou seja, era espalhada num ângulo $>90^{\circ}$. Os resultados dessas experiências de Geiger e Marsden foram examinados por Rutherford, em 191151, em experiências realizadas com o espalhamento de $\alpha$ e $\beta$ pela matéria, experiências essas que o levaram a propor seu célebre modelo planetário do átomo:

Um caroço central (núcleo, do latim nucleus, que signifca "parte interna") carregado positivamente, envolto por elétrons girando em órbitas circulares (eletrosfera), e que tornavam o átomo neutro.

Durante alguns anos, as linhas do He observadas por Lohmann eram as únicas exceções do "efeito Zeeman complexo", até que Voigt e seu aluno de doutoramento, o físico di-

\footnotetext{
${ }^{46}$ Physikalische Zeitschrift, v. 8, p. 232, 1907.

${ }^{47}$ Physikalische Zeitschrift, v. 9, p. 145, 1908.

${ }^{48}$ Proceedings of the Royal Society of London A81, p. 162, 1908.

${ }^{49}$ Proceedings of the Royal Society of London A81, p. 174, 1908.

${ }^{50}$ Proceedings of the Royal Society of London A82, p. 495, 1909.

${ }^{51}$ Proceedings of the Manchester Literary and Philosophical Society, v. 55, p. 18; Philosophical Magazine, v. 5 , p. $576 ; 21$, p. $669,1911$.
} 
namarquês Hans Marius Hansen (1886-1956), ao estudarem o efeito Zeeman inverso (separa-

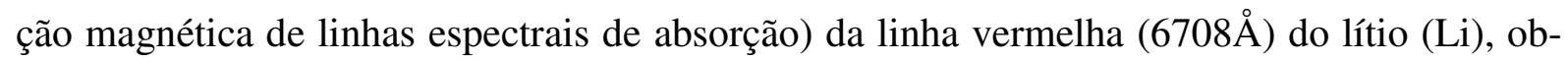
servaram em $1912^{52}$ que a mesma era separada apenas em "tripleto normal Zeeman". Observe-se que esse resultado representava uma surpresa, pois sendo o espectro do Li análogo aos do Na e potássio $(\mathrm{K})$, ele, igualmente como estes dois últimos, deveria apresentar o mesmo "efeito Zeeman complexo".

Para investigar mais apuradamente essas dificuldades com os efeitos Zeeman normal e "complexo", Paschen convidou seu aluno de doutoramento, o físico alemão Ernst Emil Alexander Back (1881-1959). Assim, em 191253, ao analisarem o Li e outros elementos químicos [p.e.: hidrogênio $(\mathrm{H})$ e He], eles descobriram que, quando o campo magnético começa a aumentar, muitos componentes magnéticos "complexos" sofrem uma "transformação magnética", tornando-se cada vez mais fracos, até se reduzirem a uma configuração "Zeeman normal". Em outras palavras, o "efeito Zeeman anômalo" (EZA) (como eles passaram a chamar, nessa ocasião, o "efeito Zeeman complexo") transforma-se no EZN, à medida que aumenta o campo magnético externo. Logo depois, em 191354, Paschen e Back confirmariam sua descoberta, ao observarem uma "transformação magnética" do oxigênio $(\mathrm{O})$, sob um campo magnético de 40 kilogauss. Assim, o efeito Paschen-Back [nome cunhado pelo físico alemão Arnold Johannes Wilhelm Sommerfeld (1868-1951), em 191455], explicou as Regras de Preston e de Runge. Ainda em 1913, Voigt ${ }^{56}$ e, independentemente, Sommerfeld ${ }^{57}$ estudaram o EZN e a "transformação magnética" [efeito Paschen-Back (EP-B)] assumindo os elétrons como sendo presos anisotropicamente aos átomos, sem, contudo, obterem bons resultados.

O modelo atômico Rutherfordiano apresentava um grande problema, pois a eletrosfera era instável, devido à radiação de Larmor. Então, para estabilizá-lo, o físico dinamarquês Niels Hendrik Bohr (1885-1962; PNF, 1922), em três artigos publicados em 191358, formulou seu modelo atômico quântico (modelo quântico de Bohr - MQB) baseado em dois postulados: 1) A energia (W) de cada elétron em uma configuração estacionária é dada pela expressão $\mathrm{W}=\omega \tau \mathrm{h} / 2$, onde $\omega$ é a frequência de revolução (angular) do elétron, $\tau$ é um número inteiro, e h é a constante de Planck; 2) A passagem dos sistemas entre diferentes configurações estacionárias é seguida pela emissão de uma radiação homogênea, para a qual a relação entre a

\footnotetext{
52 Physikalische Zeitschrift, v. 13, p. 217, 1912.

53 Annalen der Physik, v. 39, p. 897, 1912.

54 Annalen der Physik, v. 40, p. 960, 1913.

55 Königlich Gesellschaft der Wissenschaften zu Göttingen Nachrichten, p. 207, 1914.

56 Annalen der Physik, v. 40, p. 368; 41, p. 403; 42, p. 210, 1913.

57 Annalen der Physik, v. 40, p. 748, 1913.

58 Philosophical Magazine, v. 26, p. 1; 476; 857, 1913.
} 
sua frequência $(v)$ (ou comprimento de onda $\lambda$, pois $\lambda \nu=\mathrm{c}$ ) e a quantidade de energia emitida $\left(\mathrm{W}_{\tau 2}-\mathrm{W}_{\tau 1}\right)$ sendo dada por: $\mathrm{W}_{\tau 2}-\mathrm{W}_{\tau 1}=\mathrm{h} v$.

Registre-se que, com esses princípios, ele demonstrou que: 1) o momento angular (L) de um elétron em cada órbita valia: $\mathrm{L}=\tau(\mathrm{h} / 2 \pi)=\tau \hbar$, (que hoje se conhece como a regra de quantização de Bohr); e 2) a energia (E) dos elétrons em suas órbitas circulares era dada por [em eV, que representa a energia eletrostática de um elétron $\left(\mathrm{e}^{-}\right)$sob a diferença de potencial de 1 volt $(\mathrm{V})]): \mathrm{E} \approx 13,6 / \mathrm{n}^{2}$, sendo $\mathrm{n}=1,2,3, \ldots$ e conhecido como número quântico principal Bohriano. É oportuno observar que, em 191459, os físicos alemães James Franck (1882-1964; PNF, 1925) e Gustav Ludwig Hertz (1887-1975; PNF, 1925) confirmaram o MQB com suas experiências sobre a colisão entre moléculas de um gás e elétrons lentos.

O MQB foi ampliado para órbitas elípticas, em 1915, em trabalhos independentes de Sommerfeld ${ }^{60}$ e dos físicos, o japonês Jun Ishiwara (1881-1947) ${ }^{61}$ e o inglês William Wilson (1876-1965)62. Nesse modelo, conhecido então como o Modelo Atômico Quântico de BohrIshiwara-Wilson-Sommerfeld (MAQB-I-W-S), aparecem os números quânticos: $\mathrm{n}_{\mathrm{r}}$ (número quântico radial) e $\mathrm{n}_{\varphi}$ (número quântico azimutal).

Um estudo mais elaborado do EZN foi apresentado por Sommerfeld, em 191663, ao estudar o movimento (relativista e não relativista) de um elétron sob a influência de um campo magnético $\mathrm{H}$, ele introduziu um terceiro número quântico $\mathrm{m}$ (conhecido posteriormente como número quântico magnético), que determinava as posições das órbitas dos elétrons em relação à direção de $\mathrm{H}$, de tal modo que o cosseno (cos) do ângulo $\theta$ entre as direções de $\mathrm{H} \mathrm{e}$ da normal ao plano da órbita era dado por: $\cos \theta=m / n_{\varphi}$. Ora, como $m$ e $n_{\varphi}$ são números inteiros (quantizados), os valores correspondentes para $\theta$ indicavam que os planos das órbitas eletrônicas não podiam ser quaisquer e, sim, apenas os determinados por aquela condição, ou seja, tais planos variavam discretamente no espaço. Esse resultado ficou conhecido como o princípio da quantização do espaço. Ainda naquele artigo, Sommerfeld demonstrou que as linhas do espectro do hidrogênio $\left({ }_{1} \mathrm{H}^{1}\right)$ eram deslocadas de $\Delta v=\Delta v_{(\mathrm{Zn})} \times \Delta \mathrm{m}$, onde $\Delta v_{(\mathrm{Zn})}$ representava o mesmo "deslocamento Zeeman normal" que fora deduzido por Larmor e Lorentz, segundo vimos acima, e $\Delta \mathrm{m}$ media a variação de $\mathrm{m}$, variação essa que só podia assumir os valores discretos, ou seja: $\Delta \mathrm{m}=0, \pm 1, \pm 2, \ldots$ Ora, em vista dessa "regra de seleção", esse resultado de Sommerfeld apenas explicava o EZN. Por outro lado, não havia indicação nos cálculos desse trabalho de Sommerfeld de algo que se relacionasse com o EZA ou mesmo com o efeito Paschen-Back. Registre-se que essa "regra de seleção" foi demonstrada em 1918,

\footnotetext{
59 Verhandlungen der Deutschen Physikalisch Gesellschaft, v. 16, p. 457; 512, 1914.

${ }^{60}$ Sitzungsberichte Bayerischen Akademie Wissenschaften zü München, p. 425; 459, 1915.

61 Tokyo Sugaku Buturigakkakiwi Kizi, v. 8, p. 106, 1915.

62 Philosophical Magazine, v. 29, p. 795, 1915.

63 Physikalische Zeitschrift, v. 17, p. 491, 1916.
} 
em trabalhos distintos realizados pelos físicos, o polonês Adalbert Wojciech Rubinowicz $(1889-1974)^{64}$ e Bohr65.

Em 191966, Rutherford realizou a primeira transmutação química, na qual bombardeou o nitrogênio $\left({ }_{7} \mathrm{~N}^{14}\right)$ com a partícula $\alpha\left({ }_{2} \mathrm{He}^{4}\right)$ e o transformou no oxigênio $\left({ }_{8} \mathrm{O}^{17}\right)$ acompanhado do núcleo do hidrogênio $\left({ }_{1} \mathrm{H}^{1}\right):{ }_{2} \mathrm{He}^{4}+{ }_{7} \mathrm{~N}^{14} \rightarrow{ }_{8} \mathrm{O}^{17}+{ }_{1} \mathrm{H}^{1}$. Logo em $1920^{67}$, ele sugeriu que em seu núcleo atômico, além de cargas positivas [composta de ${ }_{1} \mathrm{H}^{1}$ e ao qual denominou de próton (p) (de protos, que significa "primeiro" em grego)] existiam, também, cargas neutras [nêutron (n)], descobertas mais tarde, segundo veremos mais adiante.

Muito embora o EZN tenha sido explicado por Sommerfeld (1916), no entanto, seu modelo foi insuficiente para explicar o EZA e, por isso, Sommerfeld começou a usar novos argumentos físicos para explicá-lo. Assim, em 1920, Sommerfeld voltou a essa questão com a publicação de dois trabalhos. No primeiro ${ }^{68}$, formulou a regra de decomposição magnéticoóptica que é caracterizada pela equação $\mathrm{r}=\mathrm{r}_{1} \mathrm{r}_{2}$, onde $\mathrm{r}$ é o "número de Runge" do "efeito Zeeman complexo" e $\mathrm{r}_{1}$ e $\mathrm{r}_{2}$ são também "números de Runge" dos termos envolvidos na transição eletrônica que caracteriza aquele efeito. No segundo trabalho ${ }^{69}$, Sommerfeld usou nessa sua "regra", os "números de Runge" [aos quais denominou de "números misteriosos" (Zahlemmysterium)], o MAQB-I-W-S e mais o Princípio da Combinação de Ritz [apresentado, em $1908^{70}$, pelo físico suíço Walther Ritz (1878-1909), segundo o qual a frequência de uma linha arbitrária do espectro de qualquer átomo, pode ser representada como a soma algébrica das frequências de duas outras linhas quaisquer do mesmo espectro], para explicar o "efeito Zeeman complexo".

Contudo, ainda naquele trabalho, Sommerfeld encontrou sérias dificuldades na explicação de alguns dupletos observados experimentalmente, notadamente o famoso dubleto formado pelas linhas amarelas $\left(\mathrm{D}_{1}\right.$ e $\left.\mathrm{D}_{2}\right)$ do Na. Ora, segundo o MAQB-I-W-S, o Na só poderia apresentar uma linha (singleto) $\mathrm{D}$ derivada da transição eletrônica entre a órbita $\mathrm{n}_{\varphi}=1$ [hoje, número quântico orbital ( $\ell$ )], órbita essa denominada de "onda" $\mathrm{p}$ (de principal) pelos espectroscopistas] e a órbita $\mathrm{n}_{\varphi}=0$, órbita essa denominada de "onda" $\mathrm{s}$ (de sharp) ainda pelos espectroscopistas. Desse modo, para explicar o dupleto observado do $\mathrm{Na}$, ainda naquele segundo trabalho de 1920, Sommerfeld postulou a existência de um novo número quântico, denominado por ele de "número quântico interno" e denotado por j, e que, de alguma forma, deveria estar relacionado com uma rotação "escondida" dentro do átomo.

\footnotetext{
64 Physikalische Zeitschrift, v. 19, p. 441; 465, 1918.

65 Köngelige Danske Videnskabernes Selskab Skrifter, v. 8. Laekke, IV. 1, p. 1; 37, 1918.

66 Philosophical Magazine, v. 37, p. 581, 1919.

${ }^{67}$ Proceedings of the Royal Society of London A97, p. 374, 1920.

68 Naturwissenschaften, v. 8, p. 61, 1920.

69 Annalen der Physik, v. 63, p. 221, 1920.

${ }^{70}$ Physikalische Zeitschrift, v. 9, p. 521, 1908.
} 
Esse novo número quântico (que mais tarde foi visto relacionar-se com o spin do elétron, como veremos adiante) postulado por Sommerfeld indicava que as órbitas eletrônicas elípticas deveriam ser desdobradas em outras órbitas. Assim, aos subníveis $\left(\mathrm{n}, \mathrm{n}_{\varphi}\right.$ ) (onde $\mathrm{n}$ representa o número quântico principal bohriano) do MAQB-I-W-S deveria ser incorporado um sub-subnível (j), de modo que aquelas órbitas seriam caracterizadas pelos números quânti$\cos \left(n, n_{\varphi}, j\right)$. Desse modo, para explicar outros resultados experimentais relativos a singletos, dupletos e tripletos, Sommerfeld impôs, respectivamente, que: $\mathrm{j}=\mathrm{n}_{\varphi}, \mathrm{j}=\mathrm{n}_{\varphi}, \mathrm{n}_{\varphi}-1, \mathrm{j}=\mathrm{n}_{\varphi}, \mathrm{n}_{\varphi}$ $-1, \mathrm{n}_{\varphi}-2$, além de considerar $\Delta \mathrm{j}=0, \pm 1$. É oportuno destacar que Sommerfeld foi levado a impor essa "regra de seleção" para j em virtude de haver observado que a "onda" d (de diffu$s e$, ainda segundo os espectroscopistas) do Na, que decorre da transição do subnível ( $\mathrm{n}=4, \mathrm{n}_{\varphi}$ =3) para o subnível $\left(\mathrm{n}=3, \mathrm{n}_{\varphi}=2\right)$, poderia ser um tripleto. No entanto, Sommerfeld notou, ao estudar as transições entre os dois sub-subníveis $\left(\mathrm{n}=4, \mathrm{n}_{\varphi}=3, \mathrm{j}=3\right.$ ou 2$)$ e $\left(\mathrm{n}=3, \mathrm{n}_{\varphi}=2, \mathrm{j}=\right.$ 2 ou 1), que poderia também haver o quadrupleto: $\mathrm{j}=3 \rightarrow \mathrm{j}=2, \mathrm{j}=3 \rightarrow \mathrm{j}=1, \mathrm{j}=2 \rightarrow \mathrm{j}=2 \mathrm{e}$ $\mathrm{j}=1 \rightarrow \mathrm{j}=1$. Aí, então, veio-lhe a ideia de "proibir" a transição $\mathrm{j}=3 \rightarrow \mathrm{j}=1$.

O EZA foi investigado pelo físico alemão Alfred Landé (1888-1975), em 1921, por intermédio de dois trabalhos. Assim, no primeiro deles ${ }^{71}$, considerou que o número quântico j proposto por Sommerfeld (o qual denominou de k) significava o "número quântico azimutal" do átomo como um todo (correspondente ao momento angular total do átomo), e cuja projeção na direção do campo magnético $\mathrm{H}$ era representado por um outro número quântico chamado por ele de "número quântico equatorial" e denotado por m. Desse modo, para Landé, o estado estacionário de energia (E) de um átomo em um campo $\mathrm{H}$ era dado por: $\mathrm{E}=\mathrm{E}_{1}+\mathrm{g} \mathrm{m} \mathrm{h}$ $v_{\mathrm{L}}$, onde $\mathrm{E}_{1}$ é o estado de energia do átomo não perturbado e $\mathrm{g}$ é um "fator de proporcionalidade", mais tarde conhecido como fator de Landé. Para explicar os singletos, dupletos e tripletos conhecidos, Landé então propôs que $\mathrm{m}=0, \pm 1, \pm 2, \ldots \pm \mathrm{j}$, para singletos e tripletos, e $\mathrm{m}= \pm 1 / 2, \pm 3 / 2, \ldots \pm(\mathrm{j}-1 / 2)$, para os dupletos e, mais ainda, que na transição óptica, deveríamos ter: $\Delta \mathrm{m}=0 \pm 1$. Quanto ao g, Landé propôs que ele seria unitário no caso do EZN, e assumiria valores diferentes de um $(\mathrm{g} \neq 1)$ para o EZA. No segundo trabalho ${ }^{72}$, Landé observou que, por ser $\mathrm{g} \neq 1$ no EZA, então a razão giromagnética do elétron [relação entre o momento magnético do elétron $(\mu=\mathrm{e} v \mathrm{r} / 2)$ e o seu momento angular $\left(\mathrm{L}=\mathrm{m}_{\mathrm{e}} \mathrm{v} r\right)$ em sua órbita circular de raio (r) e velocidade [v (de módulo v)] em torno do núcleo atômico] era diferente de seu valor clássico, qual seja: $\mu / \mathrm{L}=\mathrm{e} /\left(2 \mathrm{~m}_{\mathrm{e}}\right)$. Ora, como $v_{\mathrm{L}}$ é função dessa relação $\left[v_{\mathrm{L}}=\right.$ $\mathrm{e} /\left(2 \mathrm{~m}_{\mathrm{e}}\right) \times \mathrm{H} /(2 \pi \mathrm{c})$, como vimos acima], então Landé propôs que essa expressão deveria ser alterada para explicar a "anomalia" (que será tratada mais adiante) que encontrou ao estudar o movimento do elétron atômico na presença de um campo magnético externo.

O EZA também foi pesquisado pelo físico alemão Werner Karl Heisenberg (19011976; PNF, 1932). Com efeito, em 1921, ele participava dos seminários ministrados por

71 Zeitschrift für Physik, v. 5, p. 231, 1921.

72 Zeitschrift für Physik, v. 7, p. 398, 1921. 
Sommerfeld, na Universidade de Munique (UM), a respeito da Teoria das Linhas Espectrais (TLE). Em um desses seminários, Sommerfeld pediu que Heisenberg examinasse os últimos dados experimentais apresentados por Back, nesse mesmo ano de 192173, sobre o EZA, levando em consideração a teoria que Sommerfeld desenvolvera, em 1920, sobre os "números misteriosos". Depois de examiná-los, Heisenberg foi a Sommerfeld e apresentou-lhe a ideia de que os estados dupletos poderiam ser mais bem interpretados se aqueles "números" fossem considerados como semi-inteiros ao invés de inteiros, conforme Sommerfeld havia admitido. Ao saber disso, Sommerfeld ficou muito chocado e falou a Heisenberg:

- Isto é absolutamente impossível. O único fato que conhecemos sobre a teoria quântica é que existem números inteiros e não semi-inteiros.

Refeito do choque e pensando na proposta de Heisenberg, Sommerfeld convidou Heisenberg para que, no verão de 1922, o acompanhasse até Göttingen, onde Bohr iria ministrar algumas conferências e, desse modo, pudesse conhecê-lo pessoalmente para, se houvesse oportunidade, apresentar sua hipótese. Cada uma dessas conferências era seguida de longas discussões e, ao final da terceira, Heisenberg fez uma ousada observação crítica sobre o estado atual da TLE e, provavelmente, falou sobre os "números quânticos fracionários". Essa ousadia valeu-lhe um convite de Bohr para caminharem a uma montanha (a Hain) próximo de Göttingen para discutirem mais sobre a espectroscopia. Como resultado dessa caminhada, surgiu o convite de Bohr para que Heisenberg fosse à Universidade de Copenhague (UC) trabalhar com ele, pelo menos por um semestre, para que juntos pudessem "fazer um pouco de física".

A hipótese de Heisenberg sobre "números quânticos fracionários" foi desenvolvida por ele em seu primeiro trabalho científico (submetido à publicação em 17 de dezembro de 1921), publicado em $1922^{74}$, no qual apresentou o modelo de caroço ("core model") segundo o qual, em um átomo de muitos elétrons, um grande número deles circula em torno do núcleo compondo um "caroço de elétrons", enquanto os mais externos são fracamente ligados a esse mesmo núcleo, constituindo os chamados "elétrons ópticos" (ou "elétrons de valência”), que são os responsáveis pelas transições (radiação) eletrônico-ópticas bohrianas. Assim, para explicar os dupletos do EZA, nesse seu primeiro trabalho, Heisenberg postulou que o "caroço de elétrons" era dotado de um momento angular $(\mathrm{L})$ igual a $\mathrm{h} / 2$ e que o "elétron de valência" era dotado de um L igual a $\left(\mathrm{n}_{\varphi}-1 / 2\right) \hbar$, sendo que o dupleto decorria, exatamente, do alinhamento e do anti-alinhamento, respectivamente, desses momentos angulares. Note-se que a ideia de tratar um átomo de muitos elétrons, separando os mesmos em uma parte interna (" $\mathrm{ca}$ roço de elétrons") e uma parte externa ("elétrons de valência”), já havia sido utilizada pelo próprio Sommerfeld, em 191675. Por sua vez, em 192076, o físico russo Dmitry Sergeevich

\footnotetext{
${ }^{73}$ Naturwissenschaften, v. 9, p. 199, 1921.

${ }^{74}$ Zeitschrift für Physik, v. 8, p. 273, 1922.

75 Sitzungsberichte der Bayerischen Academie der Wissensschaften (München), Mathematischephysikalische Klasse, p. 131, 1916.
} 
Roschdestwensky (1876-1940) explicou a origem dos termos dupleto e tripleto como sendo devidos à ação do campo magnético produzido pelo "caroço de elétrons" sobre os "elétrons de valência", isto é, como uma espécie de "efeito Zeeman interno". Com essa hipótese ele chegou a calcular a separação das primeiras linhas da "onda" p do Li.

Depois desse primeiro trabalho, Heisenberg publicou mais três artigos, também em 1922. $\mathrm{Um}^{77}$ isoladamente, e os outros dois (com Sommerfeld) ${ }^{78}$, respectivamente, tratando dos dubletos de raios- $X$ relativísticos e definição de linha espectral e da intensidade das linhas múltiplas e seus componentes de Zeeman.

Para prosseguir com o histórico do MPPE, é importante destacar um fato novo que aconteceu no ano de 1922: a descoberta de espectros com mais de três linhas e não decorrentes de separação magnética, descoberta essa realizada, independentemente, pelo físico espanhol Miguel Antonio Catalán (1894-1957) 79 e por uma estudante de Paschen, Hilde Gieseler ${ }^{80}$ ao estudarem, respectivamente, os espectros do $\mathrm{Mg}$ e do Cr. Para explicar esses multipletos (nome cunhado por Catalán), Sommerfeld esboçou um novo modelo atômico em trabalho publicado em 192381, no qual considerou que o momento angular total (J) de um átomo, era a composição vetorial entre o momento angular total $\left(\mathrm{J}_{0}\right)$ do átomo não excitado e o momento angular $\left(\mathrm{J}_{1}\right)$ da excitação, sendo inteiros os números quânticos associados a esses dois momentos. No entanto, dificuldades com os átomos álcalis (Li, Na, etc.) levaram Sommerfeld a adicionar o momento angular proposto por Heisenberg para o "caroço de elétrons", isto é, $\mathrm{h} / 2$, tanto a $\mathrm{J}_{0}$ quanto a $\mathrm{J}_{1}$.

Esse modelo vetorial atômico proposto por Sommerfeld foi retomado por Landé, ainda em $1923^{82}$, porém com outra interpretação. Com efeito, para Landé, o J do átomo seria a soma vetorial entre o momento angular $(\mathrm{K})$ dos "elétrons de valência" e o momento angular (R) do "caroço de elétrons", ou seja: $\mathrm{J}=\mathrm{K}+\mathrm{R}$. De posse desse "modelo vetorial", Landé explicou o EZA supondo que, enquanto os vetores $\mathrm{K}$ e $\mathrm{R}$ precessionavam em torno de $\mathrm{J}$, este precessionava em torno do campo magnético externo $\mathrm{H}$. Assim, considerando que os números quânticos correspondentes aos três momentos angulares eram "números fracionários Heisenbergianos", Landé deduziu uma nova expressão para o seu "fator de proporcionalidade" (g): $\mathrm{g}=1+\left(\mathrm{j}^{2}+\mathrm{k}^{2}-\mathrm{r}^{2}\right) /\left(2 \mathrm{j}^{2}\right)$. É importante registrar que, com o desenvolvimento da Mecânica Quântica (MQ), entre 1925 e 1926 (a ser tratada mais adiante), foi visto que o valor do fator de Landé que melhor se ajustaria aos resultados experimentais seria aquele em que os quadra-

\footnotetext{
76 Transactions of the Optical Institute in Petrograd 1, p. 1, 1920.

77 Zeitschrift für Physik, v. 23, p. 363, 1922.

78 Zeitschrift für Physik, v. 10, p. 393; 11, p. 131, 1922.

${ }^{79}$ Philosophical Transactions of the Royal Society of London A223, 1922.

${ }^{80}$ Annalen der Physik, v. 69, p. 147, 1922.

81 Annalen der Physik, v. 70, p. 32, 1923.

${ }^{82}$ Zeitschrift für Physik, v. 15, p. 189, 1923.
} 
dos dos números quânticos fossem substituídos pelo produto dele por ele próprio adicionado da unidade [por exemplo: $\left.\mathrm{j}^{2}=\mathrm{j}(\mathrm{j}+1)\right]$.

Continuemos com o histórico do MPPE. No começo da década de 1920, desenvolveu-se uma questão polêmica relacionada à energia das $\beta$, pois se desejava saber se ela era determinada pelas energias dos núcleos final e inicial ou se variava continuamente, como observara Chadwick (1914). Além do mais, havia uma questão objetiva: se um elétron é emitido por um núcleo A que se transforma no núcleo $\mathrm{B}$ e tem energia menor do que as energias dos núcleos A e B, para onde vai a energia que está faltando? Em 192483, Bohr e os físicos, o holandês Hendrik Anthony ("Hans") Kramers (1894-1952) e o norte-americano John Clarke Slater (1900-1976) formularam a hipótese de que os princípios de conservação da energia e do momento linear não valiam para processos microscópicos, como o decaimento $\beta$ e o recente Efeito Compton-Debye (EC-D), descoberto pelo físico norte-americano Arthur Holly Compton (1892-1962; PNF, 1927), ao estudar o espalhamento de raios-X pelos elétrons da matéria, traduzido pela expressão: $\lambda^{\prime}-\lambda=\Delta \lambda=\left(\mathrm{h} / \mathrm{m}_{\mathrm{e}} \mathrm{c}\right)(1-\cos \theta)$, onde $\lambda^{\prime}$ e $\lambda$ representam, respectivamente, os comprimentos de onda dos raios- $X$, depois e antes de ser espalhados por elétrons de massa $m_{\mathrm{e}}$ e $\theta$ é o ângulo de espalhamento, estudo esse publicado em $1923^{84}$ e confirmado, também em $1923^{85}$, pelo físico e químico holandês Petrus Joseph Wilhelm Debye (1884-1966; PNQ, 1936)].

Apesar do grande avanço alcançado pelo Modelo Vetorial Atômico de SommerfeldLandé (MVAS-L) no sentido de explicar, quer o EZA, quer a estrutura de multipletos, novos resultados experimentais [como os "supermultipletos" observados nos espectros do néon $(\mathrm{Ne})$ e dos alcalinos terrosos: berílio (Be), cálcio $(\mathrm{Ca}), \mathrm{Mg}$ etc.] não conseguiam ser explicados por esse modelo. Em vista dessa dificuldade, esse modelo passou a ser questionado. Um dos primeiros questionamentos foi apresentado pelo físico austríaco Wolfgang Pauli Junior (19001958; PNF, 1945), em dois trabalhos realizados em $1925^{86}$, nos quais tratou relativisticamente o elétron naquele modelo e, com isso, demonstrou que os componentes Zeeman deveriam depender do número atômico $\mathrm{Z}$ do átomo considerado. No entanto, tal dependência não era conhecida experimentalmente e nem foi confirmada posteriormente; em consequência disso, Pauli inferiu que o momento angular $\mathrm{R}$, atribuído ao "caroço de elétrons" por Landé, era devido a uma nova propriedade quanto-teórica do elétron e à qual denominou uma duplicidade não descritível classicamente.

O exame do espectro de multipletos de átomos álcalis e alcalinos terrosos levou Pauli, nos dois trabalhos referidos acima, a formular o seu "modelo atômico" composto de quatro números quânticos para o elétron, assim distribuídos: o número quântico principal bohriano

\footnotetext{
${ }^{83}$ Philosophical Magazine, v. 47, p. 785; Zeitschrift für Physik, v. 24, p. 69, 1924.

${ }^{84}$ Physical Review, v. 21, p. 483, 1923.

85 Physikalische Zeitschrift, v. 24, p. 161, 1923.

86 Zeitschrift für Physik, v. 31, p. 373; 765, 1925.
} 
(n), o número quântico azimutal Sommerfeldiano $\left[\mathrm{n}_{\varphi}(\mathrm{k})\right]$ e dois números quânticos magnéticos $\left(\mathrm{m}_{1}\right.$ e $\left.\mathrm{m}_{2}\right)$, sendo que, em alguns casos, eram considerados dois $\mathrm{k}\left(\mathrm{k}_{1} \mathrm{e} \mathrm{k}_{2}\right)$ e apenas um $\mathrm{m}$ $\left(\mathrm{m}_{1}\right)$. De posse desse modelo, Pauli passou a examinar a Tabela Periódica dos Elementos e, na segunda parte do segundo trabalho de 1925, ele formulou o seu célebre Princípio da Exclusão: - Dois elétrons em um campo de força central nunca podem estar em estados de energia de ligação com os mesmos quatro números quânticos. Então, baseado nesse princípio, Pauli conseguiu distribuir os elétrons nas diversas camadas eletrônicas (níveis $\mathrm{K}, \mathrm{L}, \mathrm{M}, \mathrm{N}, \ldots$ ) e, com isso, uma primeira explicação daquela tabela foi então conseguida. Registre-se que o nome Princípio da Exclusão de Pauli foi cunhado pelo físico inglês Paul Adrien Maurice Dirac (1902-1984; PNF, 1933), em 192687.

Nessa altura em que havia uma verdadeira disputa entre os modelos atômicos, o MVAS-L e o dos "quatro números quânticos" de Pauli [cuja junção dos dois constitui a Velha Mecânica Quântica (VMQ)], é que apareceu a ideia do spin do elétron, ou seja, a ideia de que o elétron possuía uma "rotação própria". Aliás, é interessante ressaltar que a hipótese de o elétron possuir um "momento angular intrínseco" já havia sido sugerida por Compton, em $1921^{88}$, com o objetivo de explicar as propriedades magnéticas dos metais, propriedades essas que decorreriam do momento magnético $(\mu)$ do elétron associado ao "momento angular intrínseco". Porém, para Compton, esse momento angular valia ћ. No entanto, quem teve a ideia de usar esse "momento angular intrínseco" do elétron no sentido de explicar o EZA, bem como para explicar a estrutura de multipletos e supermultipletos, foi o físico alemão Ralph de Laer Krönig (1904-1995), no começo de 1925. Com efeito, para Krönig, o quarto número quântico proposto por Pauli nada mais era do que o momento angular próprio do elétron, que, contudo, valia $\mathrm{h} / 2$. Ao discutir essa sua hipótese com Pauli, na presença de Landé, Pauli com a sua proverbial atitude de reagir quase sempre contra ideias novas, disse enfaticamente para Krönig:

- Isto é, seguramente, uma ideia bastante inteligente, mas a Natureza não é assim.

Em vista dessa afirmativa, Krönig não publicou de imediato esse sua ideia, só vindo a fazê-lo em 192689, quando os físicos holandeses George Eugene Uhlenbeck (1900-1988) e Samuel Abraham Goudsmit (1902-1978) já a haviam publicado, em 1925ㅇ, em seu famoso trabalho no qual propuseram, como Krönig, associar o quarto número quântico de Pauli com uma "rotação intrínseca do elétron" [ $\operatorname{spin}(\mathrm{S})]$, e com essa hipótese explicaram vários resultados experimentais espectroscópicos, principalmente o EZA.

\footnotetext{
${ }^{87}$ Proceedings of the Royal Society of London 112A, p. 661, 1926.

88 Journal of the Franklin Institute, v. 192, p. 145, 1921.

${ }^{89}$ Nature, v. 117, p. 550, 1926.

90 Naturwissenschaften, v. 13, p. 953, 1925.
} 
É oportuno registrar que a ideia de atribuir spin ao elétron foi apenas de Uhlenbeck ao raciocinar da seguinte maneira: segundo Pauli, há quatro números quânticos caracterizando o elétron; então deve haver quatro graus de liberdade; ora, como há três graus de liberdade de translação do elétron em torno do núcleo, então o quarto grau de liberdade só poderia ser devido a uma "rotação interna" do elétron, concluiu Uhlenbeck. Goudsmit, por sua vez, não concordava com essa hipótese, pois admitia que o número quântico $\mathrm{m}_{\mathrm{S}}$ (que considerou em substituição ao $m_{1}$ de Pauli), deveria assumir apenas os valores $+1 / 2$ e $-1 / 2$. Contudo, a hipótese do spin de Uhlenbeck explicava a razão pela qual o $\mathrm{m}_{\mathrm{S}}$ de Goudsmit só poderia assumir os dois valores indicados acima.

Com relação ao spin das partículas, é interessante registrar que elas são classificadas em dois tipos: férmions de valor semi-inteiro $( \pm 1 / 2, \pm 3 / 2, \ldots)$ e que satisfazem a Estatística de Fermi (1926)-Dirac (1926); e bósons de valor inteiro $(0, \pm 1, \pm 2, \ldots)$ e que satisfazem a Estatística de Bose (1924)-Einstein (1924).

Denote-se que a comprovação experimental de $\mathrm{S}$ já havia sido obtida pelos físicos alemães Walther Gerlach (1899-1979) e Otto Stern (1888-1969; PNF, 1943), em 192291, ao realizarem uma experiência na qual observaram que um feixe de átomos de prata $(\mathrm{Ag})$, na forma de vapor e ao atravessar uma região de campo magnético variável, era separado em dois feixes. Naquela ocasião, esse resultado foi interpretado como sendo a comprovação experimental da hipótese (já referida anteriormente) da quantização espacial proposta por Sommerfeld, em 1916, uma vez que, segundo o MAQB-I-W-S, o feixe original dos átomos de Ag deveria ser separado em dois feixes, sob a ação da força $\mu_{\mathrm{z}}(=\partial \mathrm{H} / \partial \mathrm{z})$, onde $\mu_{\mathrm{z}}$ é o componente do momento magnético $(\mu)$ do elétron na direção $H$, uma vez que $\mu_{\mathrm{z}}$ só poderia ser paralelo ou antiparalelo a H. Com o desenvolvimento da Mecânica Quântica (MQ) (ver mais adiante), mostrou-se que $\mu$ e S têm a mesma direção e, desse modo, a experiência de Stern-Gerlach passou a representar a comprovação experimental do spin do elétron.

Agora, vejamos o desenvolvimento da MQ. Entre outubro de 1922 e maio de 1923, Heisenberg foi para a Universidade de Göttingen (UG) trabalhar com o físico alemão Max Born (1882-1970; PNF, 1954). Lá, ele participou dos "seminários sobre a matéria", organizados por Born, Franck e o matemático alemão David Hilbert (1862-1943), seminários esses que se iniciavam, via de regra, com Hilbert fazendo a seguinte alocução: - Senhores, como vós, desejaria que alguém pudesse dizer-me o que é, exatamente, um átomo? Nessa ocasião, havia uma questão relacionada com uma dificuldade do MAQB-I-W-S, pois este só tinha êxito quando aplicado a sistemas com um só elétron, como o átomo de hidrogênio $(\mathrm{H})$ ou o íon de hélio (He). O que aconteceria se fosse aplicado a sistemas atômicos com vários elétrons, como o caso do hélio neutro, perguntou Born? Para responder a essa pergunta, ele próprio adaptou os métodos clássicos de perturbação usados pelos astrônomos àqueles sistemas atômicos. Assim, em 192292, com a colaboração de Pauli, Born elaborou a quantização de siste-

91 Zeitschrift für Physik, v. 8; p. 110; 9, p. 349, 1922.

92 Zeitschrift für Physik, v. 10, p. 137, 1922. 
mas mecânicos comprimidos e, com seu aluno Heisenberg, em 1923, estudou as relações de fase no MQB de átomos e moléculas ${ }^{93}$ e as trajetórias eletrônicas em um átomo de hélio exci$\operatorname{tado}^{94}$.

Como os resultados dos métodos perturbativos foram razoáveis, já que explicaram alguns fatos experimentais, Born convenceu-se de que uma mudança radical nos fundamentos da Física seria necessária e que tal mudança deveria ser realizada por intermédio de um novo tipo de Mecânica. Assim, no dia 13 de junho de 1924, a Zeitschrift für Physik recebia para publicação um trabalho de Born, intitulado Über Quantenmechanik ("Sobre a Mecânica Quântica”) onde, pela primeira vez, o nome dessa nova parte de Física, tantas vezes falada nos seminários de Göttingen, recebia letra de forma, sendo esse trabalho publicado no volume 26, p. 379, daquela Revista. Novos trabalhos realizados com essa "Nova Mecânica" foram realizados por Born. Com efeito, com seu aluno, o físico alemão Ernst Pascual Jordan (19021980), em 192595, Born aplicou sua "Nova Mecânica" aos cálculos que Planck fizera, ao estudar a interação da luz com a matéria, resultando disso a existência dos quanta de energia Planckianos, em 1900, como já registramos.

Assim, Born utilizou para tal aplicação novas grandezas que chamou de quantidades de transição, verificando, então, com surpresa, que as mesmas correspondiam aos quadrados das amplitudes de vibração das fórmulas clássicas utilizadas, anteriormente, por Planck. Desse resultado, sugeriu que tais novas grandezas poderiam representar um conceito novo em Física, o das amplitudes de transição. Ao discutir essas ideias com seu aluno Heisenberg, Born achava que essas amplitudes representavam o cerne de sua MQ, faltando, apenas, determinar o tipo de Álgebra que as ligava. Um ataque de febre de feno, em junho de 1925, obrigou Heisenberg a separar-se do grupo de Born, em Göttingen, indo para a Ilha de Helgoland, no Mar do Norte, onde começou a desenvolver suas próprias ideias sobre a MQ formulada por Born. Como o cálculo dos níveis de energia do hidrogênio, por intermédio do MAQB-I-W-S, baseava-se em quantidades aparentemente inobserváveis, como a posição e o período de revolução de um elétron em determinada órbita, Heisenberg passou a desenvolver um novo formalismo quântico teórico, envolvendo, apenas, quantidades observáveis de um átomo, como, por exemplo, os seus níveis de energia. Para chegar a esse formalismo, Heisenberg substituiu os coeficientes de Fourier da Teoria Clássica da Radiação Planckiana, e que representavam as amplitudes de radiação, por esses seus entes matemáticos que dependem dos números quânticos $(\mathrm{n}, \mathrm{m})$ característicos dos níveis de energia envolvidos na radiação. Esses novos entes foram arranjados em uma tabela (array) de $\mathrm{n}$ linhas e de $\mathrm{m}$ colunas, sendo os elementos diagonais dessa tabela relacionados com os estados estacionários, e os nãodiagonais, com as amplitudes de transição entre estados diferentes. Além do mais, Heisen-

\footnotetext{
93 Zeitschrift für Physik, v. 14, p. 44, 1923.

94 Zeitschrift für Physik, v. 16, p. 229, 1923.

95 Zeitschrift für Physik, v. 33, p. 479; 34, p. 858, 1925.
} 
berg mostrou que o produto desses novos entes gozava da propriedade não-comutativa. Esse produto era a "multiplicação simbólica" que Born tanto almejava para suas amplitudes de transição.

No dia 11 (ou 12) de julho de 1925, Heisenberg entregou a versão final desse trabalho a Born, pedindo-lhe sua opinião. Ao lê-lo, Born percebeu que o fundamento matemático do formalismo que Heisenberg utilizara, era o Cálculo Matricial que o matemático inglês Arthur Cayley (1821-1895) inventara em 185896, e que Born conhecia. Ao repetir os cálculos de Heisenberg, usando para isso a notação matricial, Born só conseguiu calcular os elementos diagonais da matriz, inferindo, por intuição, que os não-diagonais seriam nulos, já que não conseguira demonstrar tal resultado. Ao encontrar-se com Pauli (seu ex-aluno e colaborador), em uma viagem de trem, de Göttingen para Hannover, para participar de um encontro da Sociedade Germânica de Física, tentou convencer seu colaborador para fazerem um trabalho juntos com o fim de calcularem os elementos não-diagonais das matrizes que apareciam no formalismo de Heisenberg, recebendo de Pauli a seguinte resposta:

- Sim, eu sei que você gosta de formalismos tediosos e complicados. Você vai desperdiçar as ideias físicas de Heisenberg com essa matemática fútil...97

Convencido de sua ideia, Born pediu a Jordan que fizesse referidos cálculos. Depois de alguns dias Jordan voltou com os cálculos, mostrando que a matriz deveria ser diagonal devido às equações canônicas do movimento do elétron. Assim, no dia 27 de setembro de 1925, a Zeitschrift für Physik (e publicado no volume 34, p. 858) recebia para publicação o célebre trabalho de Born e Jordan, intitulado Zur Quantenmechanik ("Sobre a Mecânica Quântica"), em que o formalismo quântico Heisenbergiano é todo desenvolvido com o auxílio do Cálculo Matricial, razão pela qual essa "Nova Mecânica" passou a ser conhecida como Mecânica Quântica Matricial (MQM). O complemento dessa teoria, isto é, sua extensão a sistemas com diversos graus de liberdade, bem como a utilização da teoria da perturbação a sistemas não-degenerados e degenerados, com aplicação à Teoria de Planck do corpo negro, foi formulada por Born, Heisenberg e Jordan, em trabalho intitulado Zur Quantenmechanik II (“Sobre a Mecânica Quântica II"), e publicado no volume 35 da Zeitschrift für Physik, p. 557, no ano de 1926.

É interessante observar que Dirac, em novembro de $1925^{98}$, encontrou uma nova formulação da Mecânica Quântica de Born-Heisenberg-Jordan (MQB-H-J) por intermédio de uma relação entre a Mecânica Quântica de Born (1924) e a Mecânica Clássica de Laplace (1782)-Hamilton (1835)-Jacobi (1837) (MCL-H-J), usando o conceito de bracket ("parêntesis" - $\{\mathrm{p}, \mathrm{q}\})$ definido pelo matemático francês Siméon Denis Poisson (1781-1840), em 1809 (Journal de l'École Polytechnique 8, p. 266), e substituindo o bracket \{p, q\} pelo comutador

\footnotetext{
96 Philosophical Transactions of the Royal Society of London 148, p. 17, 1858.

97 BASSALO, J. M. F.; CARUSO, F. Pauli. Livraria da Física, 2013.

98 Proceedings of the Royal Society of London A109, p. 642, 1925.
} 
[p, q] (= pq $-\mathrm{qp})$ multiplicado por $2 \pi \mathrm{i} / \mathrm{h} 1$, sendo $\mathrm{p}$ (momento) e q (posição), operadores correspondentes às grandezas canonicamente conjugadas da MCL-H-J, sendo 1 o operador unitário e $\mathrm{i}=\sqrt{-1}$.

Ainda na primeira metade da década de 1920, ocorreu outro resultado importante para o desenvolvimento da MQ. Trata-se do seguinte. Nos primeiros vinte e cinco anos do Século 20, um dos problemas mais intrigantes da Física era o caráter dual da luz. [proposto pelo físico germano-suíço-norte-americano Albert Einstein (1879-1955; PNF, 1921)], em 191699], uma vez que em certos fenômenos ela se apresentava como onda, caráter esse observado nas experiências de difração e interferência, e em outros fenômenos se apresentava como corpúsculo, caráter esse observado no espalhamento da luz pela matéria, como no EC-D.

O problema do caráter dual da luz referido acima fascinou o físico francês, o Príncipe Louis Victor Pierre Raymond de Broglie (1892-1987; PNF, 1929), que passou a estudá-lo. Assim, usando a Analogia Mecânico-Óptica (base da MCL-H-J), ele estendeu o caráter dual da luz para o elétron e para toda a matéria em geral. Vejamos como. Ao observar as órbitas dos elétrons no MQB, de Broglie verificou que a estabilidade das mesmas envolvia números inteiros, fato esse que é característico, apenas, de fenômenos de interferência e de modos normais de vibração de uma corda fixa. Portanto, considerando esse caráter dual da luz, de Broglie formulou a hipótese de que o movimento do elétron, de massa $\mathrm{m}_{\mathrm{e}} \mathrm{e}$ velocidade $\mathrm{v}$, em uma órbita circular atômica, é guiado por uma onda-piloto, cujo comprimento de onda $\lambda$ se relaciona com o seu momento linear $\left(\mathrm{p}=\mathrm{m}_{\mathrm{e}} \mathrm{v}\right)$ da mesma maneira como acontece com o quantum de luz, ou seja: $\mathrm{p}=\mathrm{m}_{\mathrm{e}} \mathrm{v}=\mathrm{h} / \lambda$. Ao considerar que esta "onda-piloto" é uma onda estacionária, ou seja, que cada órbita circular do elétron, de raio r e momento angular L, deve conter um número inteiro $(\tau)$ de "ondas-piloto", de Broglie demonstrou a regra de quantização de Bohr $(\mathrm{L}=\tau \hbar)$. Registre-se que quando de Broglie formalizou suas ideias sobre a onda de matéria ${ }^{100}$ e apresentou-as, ainda em 1924, como Tese de Doutoramento, intitulada Recherche sur la Théorie des Quanta ("Pesquisa sobre a Teoria dos Quanta"), à Faculdade de Ciências da Universidade de Paris (Sorbonne), houve um certo embaraço por parte dos professores que iriam julgá-la, uma vez que essa Tese fugia aos cânones tradicionais da Física. Assim, ela foi encaminhada ao físico francês Paul Langevin (1872-1946) para julgamento. De imediato, ele enviou uma cópia ao seu amigo Einstein que, por sua vez, pediu a Born uma opinião séria sobre a mesma, escrevendo-lhe:

- Leia isto! Embora pareça ter sido escrito por um louco, está escrito corretamente.

Quando Einstein devolveu a Tese de de Broglie a Langevin, disse-lhe que podia aprová-la, já que a mesma continha muitas descobertas importantes.

\footnotetext{
${ }^{99}$ Mitteilungen der Physikalischen Gesellschaft Zürich, v. 16, p. 47, 1916.

100 Publicadas nas Comptes Rendus de l’Académie des Sciences de Paris, v. 177, p. 507; 548; 630, 1923; 179, p. 39, 1924.
} 
A "onda de matéria deBroglieana" rapidamente "espalhou-se" pela Europa, chegando até à Universidade de Zürich, onde trabalhava o físico austríaco Erwin (Rudolf Josef Alexander) Schrödinger (1887-1961; PNF, 1933) que, impressionado pela ideia de de Broglie, começou a desenvolver uma teoria sobre a "onda de matéria", culminando com a proposição de uma equação diferencial que a mesma deveria satisfazer: a hoje mundialmente conhecida Equação de Schrödinger (ES), dada pela expressão: $H \psi(\mathrm{r}, \mathrm{t})=\mathrm{i} \hbar \partial \psi(\mathrm{r}, \mathrm{t}) / \partial \mathrm{t}$, onde $H$ é o operador hamiltoniano [soma do operador energia cinética $(L)$ com o operador energia potencial $(V)$ ] e i h $\partial$ )/Ot é o operador energia total $(E)$ de um sistema físico estacionário. Essa equação foi desenvolvida, em $1926^{101}$, no bojo de uma outra MQ, diferente da MQB-H-J, e que logo passou a ser conhecida como Mecânica Ondulatória de Schrödinger (MOS). Notese que, apesar do grande sucesso da MOS, já que a mesma explicava vários resultados experimentais, a função de onda $[\psi(\mathrm{r}, \mathrm{t})]$ ou campo escalar de sua equação carecia de uma interpretação física. Nos artigos de Schrödinger referidos acima, vê-se que a primeira tentativa fora feita por ele próprio ao interpretar os elétrons como pacotes de onda deslocando-se no espaço como se fossem partículas clássicas. Essa interpretação malogrou, pois logo depois ficou demonstrado que o "pacote" dissipava-se com o decorrer do tempo. De outra feita, ainda Schrödinger propôs que seu campo escalar poderia medir a espessura da camada formada pelo elétron "derramado" sem, no entanto, conseguir grande êxito.

Ora, como $\psi(\mathrm{r}, \mathrm{t})$ é uma função complexa, então qualquer grandeza observável [posição (r), energia (E), momento linear (p), momento angular (L) etc.] não tem significado físico e, desse modo, $\psi(\mathrm{r}, \mathrm{t})$ precisava de outra interpretação, a qual foi dada por Born, também em 1926102, que a considerou como uma onda(amplitude) de probabilidade, hipótese essa resultante de seu estudo sobre a dispersão de elétrons (representado por uma onda deBroglieana) por um átomo, no qual verificou que o número de elétrons difundidos poderia ser calculado por intermédio de certa expressão quadrática [dada por: $\psi^{*}(\mathrm{r}, \mathrm{t}) \times \psi(\mathrm{r}, \mathrm{t}) \equiv \mid \psi(\mathrm{r}$, t) $\left.\right|^{2}|\psi(\mathrm{r}, \mathrm{t})|^{2}$ e denominada por ele de probabilidade], construída a partir da amplitude da onda esférica secundária, onda essa gerada pelo átomo espalhador do feixe eletrônico incidente. É interessante destacar que, para um sistema estacionário $(\mathrm{E}=\mathrm{cte}$.), tem-se: $\psi(\mathrm{r}, \mathrm{t})=\varphi$ $(\mathrm{r}) \times \exp [-(\mathrm{i} / \mathrm{h}) \mathrm{E} \mathrm{t}], \operatorname{com} \varphi(\mathrm{r})$ sendo uma função real. Então, de posse desse resultado, virá: $\psi^{*}(\mathrm{r}, \mathrm{t}) \times \psi(\mathrm{r}, \mathrm{t}) \equiv|\psi(\mathrm{r}, \mathrm{t})|^{2}=|\varphi(\mathrm{r})|^{2}$, e que representa um valor real (portanto, medível).

A interpretação de Born vista acima ficou conhecida como Interpretação de Copenhague (IC), pois foi considerada como sendo a verdadeira por parte de Bohr, que liderava um grupo de pesquisa na Universidade de Copenhague (UC). Pois bem, a essa interpretação, sobrepôs-se outra questão. Será sempre possível observar qualquer grandeza física? A resposta a essa pergunta foi dada por Heisenberg. Vejamos como ela aconteceu. Ao tentar representar, matematicamente, a trajetória de um elétron em uma câmara de névoa ou câmara de Wil-

101 Annalen der Physik, v. 79, p. 361; 489; 734; 747; 80, p. 437; 81, p. 109; Physical Review, v. 28, p. 1049, 1926.

102 Zeitschrift für Physik, v. 37, p. 863; 38, p. 803, 1926. 
son (CW) [inventada pelo físico inglês-escocês Charles Thomson Rees Wilson (1869-1959; PNF, 1927), em 1911103], Heisenberg percebeu que, embora se observe essa trajetória por intermédio de gotinhas de água isoladas na câmara, tais gotinhas, certamente, eram muito mais amplas que um elétron e, desse modo, só se registra uma sucessão discreta de lugares, imprecisamente determinados, do elétron. Portanto, a verdadeira questão, concluiu Heisenberg, era a de representar, dentro da MQO, uma situação que, de modo aproximado - quer dizer, com certa imprecisão -, possua uma determinada velocidade. Foi, basicamente, esse raciocínio que o levou a apresentar, em 1927104, seu famoso Princípio da Incerteza (PI), assim enunciado:

É impossível obter exatamente os valores simultâneos de duas variáveis, a não ser dentro de um limite mínimo de exatidão.

É interessante anotar que, no caso das variáveis serem o momento $\left(\mathrm{p}_{\mathrm{x}}\right)$ e a posição (x), Heisenberg usou as funções $\psi(\mathrm{r}, \mathrm{t})$ de Schrödinger e a interpretação probabilística de Born e demonstrou que (em notação atual, onde $<>$ significa valor médio): $<\left(\Delta \mathrm{p}_{\mathrm{x}}\right)>$. $<(\Delta \mathrm{x})$ $>\geq \hbar / 2^{105}$.

Ainda em 1927106, Dirac publicou dois trabalhos nos quais considerou $\psi(\mathrm{r}, \mathrm{t})$ e sua conjugada $\psi^{*}(\mathrm{r}, \mathrm{t})$ como operadores (em vez de números como Schrödinger havia considerado, em 1926, como vimos acima), porém sua álgebra era não-comutativa, isto é: $\psi \psi^{*} \neq \psi^{*} \psi$. Com esse procedimento, conhecido como Teoria Quântica da Emissão e Absorção da Radiação e que ficou conhecida como Segunda Quantização, que considera os operadores: criação $\left(\mathrm{a}^{+}\right)$, destruição (a) e número de ocupação ou conservação $\left(\mathrm{N}=\mathrm{a}^{+} \mathrm{a}\right)$ que satisfazem as seguintes regras de comutação: $\left[\mathrm{a}_{\alpha}, \mathrm{a}_{\beta}^{+}\right]=\delta_{\alpha \beta}$, $[\mathrm{a}, \mathrm{a}]=\left[\mathrm{a}^{+}, \mathrm{a}^{+}\right]=0$, Dirac quantizou o campo eletromagnético, procedimento esse que deu origem ao desenvolvimento da Eletrodinâmica Quântica (QED: Quantum Electrodynamics). Destaque-se que, segundo esta teoria, no átomo de H, o elétron é preso ao próton, por intermédio da troca de uma nuvem de fótons.

Observe-se que, por ocasião da Quinta Conferência de Solvay que aconteceu em Bruxelas, também em 1927, Dirac encontrou-se com Bohr que lhe perguntou em que estava trabalhando. Dirac então lhe respondeu que buscava uma teoria relativista do elétron. Bohr retrucou dizendo-lhe que o físico sueco Oskar Benjamin Klein (1894-1977), em 1926107, já havia realizado essa teoria. Dirac não concordou com essa afirmação, pois sabia que Klein fizera apenas uma versão relativística da ES. Dirac, contudo, buscava outro caminho e que foi

\footnotetext{
103 Proceedings of the Royal Society of London A85, p. 285, 1911.

104 Zeitschrift für Physik, v. 43, p. 172, 1927.

105 BASSALO, J. M. F.; CARUSO, F. Heisenberg. Livraria da Física, 2014.

106 Proceedings of the Royal Society A114, p. 243; 710, 1927.

107 Zeitschrift für Physik, v. 37, p. 895, 1926.
} 
encontrado por ele, em 1928108, deduzindo a hoje famosa Equação de Dirac (ED) - (i $\hbar \gamma^{\mu} \partial_{\mu}$ - m c) $\Phi=0$-, na qual $\gamma^{\mu}$ é a matriz de Dirac (matriz $\left.4 \times 4\right), \partial_{\mu}=\partial / \partial \mathrm{x}^{\mu}(\operatorname{com} \mu=1,2,3,4), \Phi$ é o spinor de Dirac (matriz coluna) e m, a massa do elétron. Mais tarde, em 1930109, Dirac considerou que o m que aparece em sua equação, era uma média entre a massa do próton (p) e a massa do elétron (e).

Um resultado importante da ED foi o conceito de antimatéria. Com efeito, ao resolver essa equação e usando a expressão relativista da energia: $E^{2}=p^{2} c^{2}+m^{2} c^{4}$, Dirac encontrou que ela não só descrevia o elétron com momento p e energia positiva $(E>0)$, mas tinha outra solução que descrevia partículas idênticas a elétrons, porém com "carga positiva" e energia negativa $(\mathrm{E}<0)$. Ele então chamou essas partículas de "buracos" e afirmou que eles ocupavam todos os estados de energia negativa, o famoso "mar de Dirac". Nessa época, Dirac não havia entendido bem essa outra solução. Assim, esse "buraco" foi interpretado como sendo um próton, em 1929110, pelo matemático alemão Hermann Weyl (1885-1955) e, ainda em $1929^{111}$ e em 1930112, pelo próprio Dirac. Essa interpretação decorria do fato de que, naquela época, só se conheciam dois tipos de partículas elementares: elétrons e prótons. Por sua vez, o núcleo atômico era considerado formado apenas desses dois tipos de partículas. Porém, Dirac não ficou muito satisfeito com essa proposta, uma vez que já se sabia que p tinha massa cerca de 1.840 vezes maior do que à de $\mathrm{e}^{113}$.

Ainda em 1930, em trabalhos independentes, os físicos, o norte-americano Julius Robert Oppenheimer (1904-1967)114 e o russo Igor Yevgenyevich Tamm (1895-1971; PNF, 1958) ${ }^{115}$, mostraram que o "buraco" não poderia ser um próton, pois, desse modo, tornaria o átomo instável por causa do processo: próton + elétron $\rightarrow$ radiação luminosa $(\gamma)$ [sendo esta cunhada como fóton (do grego photo, que significa "luz"), pelo químico norte-americano Gilbert Newton Lewis (1875-1946), em 1926116]. Em 1931117, Dirac aceitou a ideia de que o "buraco" seria uma nova espécie de partícula, até então desconhecida pelos físicos experimentais, e a chamou de antielétron. Anote-se que, ainda na sequência do histórico do MPPE, registremos resultados importantes para o mesmo. Por exemplo, a explicação física do decaimento alfa (emissão de ${ }_{2} \mathrm{He}^{4}$ por núcleos radioativos, como já anotamos antes) só foi conseguida por

\footnotetext{
108 Proceedings of the Royal Society A117, p. 610; A118, p. 351, 1928.

109 Proceedings of the Cambridge Philosophical Society 26, p. 361, 1930.

110 Zeitschrift für Physik, v. 56, p. 330, 1929.

111 Proceedings of the Royal Society of London A126, p. 360, 1929.

112 Nature, v. 126, p. 605, 1930.

113 BASSALO, J. M. F.; CARUSO, F. Dirac. Livraria da Física, 2013.

114 Physical Review, v. 35, p. 562, 1930.

115 Zeitschrift für Physik, v. 62, p. 545, 1930.

116 Nature, v. 118, p. 874, 1926.

117 Proceedings of the Royal Society of London A133, p. 60, 1931.
} 
intermédio da MQO, explicação essa apresentada em 1928, pelos físicos, o norte-americano Edward Uhler Condon (1902-1974) e o inglês Ronald Wilfrid Gurney (1898-1953) ${ }^{118}$ e, independentemente, pelo russo-norte-americano George Antonovich Gamow (1904-1968) ${ }^{119}$, ao resolverem o problema daquele decaimento, usando o efeito túnel (tunelamento quântico), segundo o qual uma partícula pode vencer uma barreira de potencial, com energia menor que a do pico da barreira. Nessa formulação, eles conseguiram, inclusive, estimar a $\mathrm{T}_{1 / 2}$ dos elementos radioativos e, dessa maneira, iniciar uma nova disciplina da Física: a Física Nuclear.

Por outro lado, a polêmica sobre a conservação da energia no decaimento $\beta$, registrada anteriormente, foi resolvida por Pauli ao escrever, em 04 de dezembro de 1930, uma carta aos físicos, a sueco-austríaca Lise Meitner (1878-1968) e o alemão Geiger, que participavam da reunião do Group of Radioactivity of Tübingen. Nessa carta (intitulada: - To Radioactivity Ladies and Gentlemen), ele propunha a existência de uma partícula neutra, de massa muito pequena, não excedendo um centésimo da massa do próton, emitida junto com o elétron no decaimento $\beta$. Ainda para Pauli, o núcleo atômico era constituído de prótons, elétrons e suas partículas que, inclusive, apresentava propriedades magnéticas, isto é: seria um "nêutron magnético". Note-se que essa proposta foi também apresentada por Pauli na Reunião da Sociedade Americana de Física, realizada em junho de 1931, em Pasadena, assim como na Ann Arbor Summer School ("Escola de Verão de Ann Arbor"), na Universidade de Michigan, e publicada ainda em 1931'120. Logo depois, em 1932, o antielétron foi descoberto pelo físico norte-americano Carl David Anderson (1905-1991; PNF, 1935)121, que lhe deu o nome de pósitron $\left(\mathrm{e}^{+}\right)$. Ainda naquele ano, o nêutron $\left({ }_{0} \mathrm{n}^{1}\right)$ foi descoberto por Chadwick ${ }^{122}$. Em $1933^{123}$, os físicos, o inglês Patrick Maynard Stuart Blackett (1897-1974; PNF, 1948) e o italiano Giuseppe Pablo Stanislao Occhialini (1907-1993) realizaram uma experiência na qual confirmaram a existência do pósitron $\left(\mathrm{e}^{+}\right)$. Essa experiência, realizada no Cavendish Laboratory, na Inglaterra, hoje conhecida como produção de pares $\left(\gamma \rightarrow \mathrm{e}^{-}+\mathrm{e}^{+}\right)$, foi confirmada, ainda em $1933^{124}$, pelo físico alemão Max Delbrück (1906-1981), ao estudar o espalhamento de fótons $(\gamma)\left[\mathrm{E}>1,02 \mathrm{MeV}\left(1 \mathrm{MeV}=10^{6} \mathrm{eV}\right)\right]$ por campos eletrostáticos, como, por exemplo, o de um núcleo atômico que é carregado positivamente; esse processo é o conhecido espalhamento de Delbrück. É oportuno observar que, nesse tipo de espalhamento, a produção de pares é dita virtual, pois logo que o par é formado, ele desaparece produzindo um par de fótons $\left(\mathrm{e}^{-}+\mathrm{e}^{+} \rightarrow\right.$ $2 \gamma$ ), num processo conhecido como aniquilamento.

\footnotetext{
118 Nature, v. 122, p. 439, 1928.

119 Zeitschrift für Physik, v. 51, p. 204, 1928.

120 Physical Review, v. 38, p. 579, 1931.

${ }^{121}$ Proceedings of the Royal Society of London A41, p. 405; Science, v. 76, p. 238, 1932.

122 Proceedings of the Royal Society of London A136, p. 696; 735; Nature, v. 129, p. 312, 1932.

123 Proceedings of the Royal Society of London A139, p. 699, 1933.

124 Zeitschrift für Physik, v. 84, p. 144, 1933.
} 
Analisaremos, agora, outros processos radioativos decorrentes de experiências realizadas pelo casal Joliot-Curie [os físicos franceses, Irène (1897-1956) e Jean Frédéric JoliotCurie (1900-1958)], envolvendo colisão de partículas $\alpha$ com alvos fixos e que levou à descoberta da radioatividade artificial realizada por aquele casal. Com efeito, em janeiro de 1934, o casal bombardeou o $\mathrm{A} \ell$ com $\alpha$ e, após removerem a fonte dessas partículas, esse casal observou que o alvo daquele metal, depois de expelir nêutrons, continuava a emitir "radiações estranhas". Assim, ainda em 1934125, eles interpretaram essas "radiações" como provenientes do isótopo do fósforo $\left(\mathrm{P}^{30}\right)$, não encontrado na natureza, e que acabara de se formar em decorrência de uma reação nuclear do tipo (em notação atual): ${ }_{2} \mathrm{He}^{4}+{ }_{13} \mathrm{~A} \ell^{27} \rightarrow{ }_{15} \mathrm{P}^{30}+{ }_{0} \mathrm{n}^{1}$ com ${ }_{15} \mathrm{P}^{30} \rightarrow{ }_{14} \mathrm{Si}^{30}+\mathrm{e}^{+}\left(\mathrm{T}_{1 / 2}=3,25 \mathrm{~min}\right)$. Os isótopos radioativos artificiais assim formados passaram a denominar-se de radioisótopos.

Por sua vez, a partícula Pauliana foi denominada de neutrino ("nêutron pequenino", em italiano, e denotada por $v$ ) por Fermi, em 1934126, por ocasião em que formulou a teoria matemática do decaimento $\beta$, segundo a qual, por intermédio de uma nova força na natureza chamada mais tarde de força (interação) fraca (fr) - o nêutron (n) transforma-se em um próton (p), com a emissão de um elétron (e $\left.\mathrm{e}^{-}\right)$e da partícula Pauliana ( $v$ : hoje $\bar{v}_{e}$ ), ou seja: $\mathrm{n} \rightarrow \mathrm{p}$ $+\mathrm{e}^{-}+v\left(\bar{v}_{e}\right)$.

É interessante ressaltar que nas "radiações estranhas" que apareceram nas experiências realizadas pelo casal Joliot-Curie, estavam envolvidos processos de desintegração (em notação atual): beta-menos $\left(\beta^{-}\right)$[o nêutron (n) desintegrando-se em um próton (p), com a emissão de um elétron ( $\left.\mathrm{e}^{-}\right)$e de seu antineutrino associado $\left(\bar{v}_{e}\right)$; beta-mais $\left(\beta^{+}\right)$(beta-inverso) [o próton (p) desintegrando-se em um nêutron (n), com a emissão de um pósitron $\left(\mathrm{e}^{+}\right)$e de seu neutrino associado $\left(v_{\mathrm{e}}\right)$ ]; e a captura eletrônica [captura de um elétron ( $\mathrm{e}^{-}$) da camada $\mathrm{K}$ pelo próton (p) de um núcleo atômico, com a formação de um nêutron (n) e a emissão de um neutrino associado ao elétron $\left(v_{\mathrm{e}}\right)$ ], conforme foram interpretadas, ainda em 1934, em trabalhos independentes realizados pelo físico italiano Gian Carlo Wick (1909-1992)127 e pelos físicos, o germano-norte-americano Hans Albrecht Bethe (1906-2005; PNF, 1967) e o inglês Rudolf Ernest Peierls (1907-1995)128 e traduzidas pelas seguintes reações nucleares: $\mathrm{p} \rightarrow \mathrm{n}+\mathrm{e}^{+}+v_{\mathrm{e}}$ $\leftrightarrow \mathrm{e}^{-}+\mathrm{p} \rightarrow \mathrm{n}+v_{\mathrm{e}}$

A descoberta do n por Chadwick (1932) como uma das partículas constituintes do núcleo atômico Rutherfordiano, juntamente com o p, provocou uma grande dificuldade para os físicos, qual seja, a de explicar a razão de os prótons não se repelirem pela força Coulombiana [eletromagnética (fem)] no interior do núcleo. Para resolver essa dificuldade, ainda em

\footnotetext{
125 Comptes Rendus l’Académie des Sciences de Paris 198, p. 254; 559, Nature 133, p. 201, 1934.

126 Ricerca Scientifica, v, 4, p. 491; 1933; Nuovo Cimento, v. 11, p. 1; Zeitschrift für Physik, v. 88, p. 161, 1934.

127 Atti Rendiconti Lincei. Accademia Nationale dei Lincei 19, p. 319, 1934.

128 Nature, v. 133, p. 532, 1934.
} 
1932, Heisenberg ${ }^{129}$, o físico russo Dimitrij Iwanenko (1904-1994) ${ }^{130}$, além do físico italiano Ettore Majorana (1906-1938) (sem publicar) propuseram a hipótese de que os prótons e os nêutrons enquanto partículas constituintes do núcleo atômico se comportavam como partículas únicas - os núcleons [nome cunhado, em 1941131, pelo físico dinamarquês Christian Möller (1904-1980)] - que interagiam por intermédio de uma força atrativa capaz de superar a repulsão Coulombiana. Depois de uma polêmica entre Heisenberg e Majorana sobre a explicação dessa "força atrativa", ela foi finalmente explicada, em 1935132, quando o físico japonês Hideaki Yukawa (1907-1981; PNF, 1949) propôs que aquela "força" decorria da troca entre eles (núcleons) da partícula $U$ (como a denominou Yukawa), porém sua massa deveria ser $\mathrm{m}_{\mathrm{u}}=200 \mathrm{~m}_{\mathrm{e}}$. Para chegar a esse valor, Yukawa admitiu que a energia potencial $\mathrm{V}$ de dois núcleons em repouso seria dada pela expressão: $\mathrm{V}=-(\mathrm{A} / \mathrm{r}) \exp (\mu \mathrm{r})$, onde A é uma constante e $\mu=\mathrm{m}_{\mathrm{U}} \mathrm{c} / \mathrm{h} \approx 10^{13} \mathrm{~cm}^{-1}$. Portanto, para Yukawa, a força nuclear [mais tarde chamada de força (interação) forte (fo)] era de curto alcance e mediada (em analogia com a troca de fótons entre elétron e próton no átomo de $\mathrm{H}$, conforme visto acima) por uma partícula de massa intermediária $\left(\mathrm{m}_{\mathrm{U}}\right)$ entre a massa do elétron $\left(\mathrm{m}_{\mathrm{e}}\right)$ e a massa do próton $\left(\mathrm{m}_{\mathrm{p}}\right)$, razão pela qual a mesma ficou conhecida, inicialmente, como yukon. A primeira delas foi detectada em 1937, em experiências independentes realizadas pelos físicos norte-americanos Anderson e Seth Henry Neddermeyer (1907-1988) ${ }^{133}$; e Jabez Curry Street (1906-1989) e Edward Carl Stevenson (n.1907) ${ }^{134}$, ao anunciarem a descoberta de uma nova partícula fortemente ionizante e com massa $\left(\approx 200 \mathrm{~m}_{\mathrm{e}}\right.$ ), denominada de "elétron-pesado" (nome dado por Anderson e Neddermeyer). Um mês depois (ainda em 1937), essa descoberta foi imediatamente examinada por Oppenheimer e pelo físico norte-americano Robert Serber (1909-1997) ${ }^{135}$, ocasião em que sugeriram que essa partícula, posteriormente conhecida como o méson andersoniano ou mesotron, pois possuía uma massa intermediária [hoje: méson (do grego meso, que significa "médio") termo cunhado, em 1939136, por parte do físico indiano Homi Jehangir Bhabha (1909-1966)], entre o elétron e o próton, seria a prevista por Yukawa. É oportuno registrar que a existência dessa partícula foi confirmada nas experiências realizadas, em 1947, pelos físicos, o brasileiro Cesare (César) Mansueto Giulio Lattes, os ingleses Hugh Muirhead (1925-2007) e Sir Cecil Frank Powell (1903-1969; PNF, 1950), e o italiano Occhialini, nas quais observaram que a incidência de raios cósmicos (RC) em emulsões nucleares colocadas

\footnotetext{
129 Zeitschrift für Physik, v. 77, p. 1, 1932.

130 Nature, v. 129, p. 798, 1932.

131 Köngelige Danske Videnskab Selskab Matematisk-Fysiske Meddelanden, v. 18, p. 3, 1941.

132 Proceedings of the Physical Mathematics Society of Japan 17, p. 48, 1935.

133 Physical Review, v. 51, p. 884, 1937.

134 Physical Review, v. 51, p. 1005, 1937.

135 Physical Review, v. 51, p. 1113, 1937.

136 Nature, v. 143, p. 276, 1939.
} 
nos Alpes (Suíça) e em Chacaltaya (Bolívia) produzia dois tipos de mésons: primários [hoje: múons $(\mu)$ ] e secundários [hoje: píons $\left(\pi^{0, \pm}\right)$, e que foram publicadas, ainda em 1947, por Lattes, Muirhead, Occhialini e Powell ${ }^{137}$, e por Lattes, Occhialini e Powell ${ }^{138}$.

Ainda em 1947139, os físicos ingleses George Dixon Rochester (1908-2001) e Clifford Charles Butler (1922-1999) anunciaram que haviam descoberto uma nova partícula ao examinarem cerca de 5.000 fotografias de experiências que realizaram sobre a penetração de raios cósmicos em câmaras de Wilson $(\mathrm{CW})$ colocadas em grandes altitudes. Nesse exame, descobriram trajetórias em forma de $\mathrm{V}$ decorrentes de uma origem comum e interpretaramnas como traços deixados por partículas carregadas e provenientes da desintegração de uma partícula neutra e desconhecida, à qual deram o nome de partícula $V$, devido à forma das trajetórias observadas. Novas experiências de Rochester e Butler, ainda em 1947, evidenciaram outras partículas do tipo $\mathrm{V}$, descarregadas e carregadas $\left(\mathrm{V}^{0}, \mathrm{~V}^{ \pm}\right)$, e descritas apenas em $1953^{140}$. Note-se que essas partículas tipo $\mathrm{V}$ foram chamadas de estranhas por que eram produzidas por interação forte, entre píons $\left(\pi^{0, \pm}\right)$ e núcleons $(\mathrm{p}, \mathrm{n})$, e decaiam por interação fraca. É interessante observar que, como as $\mathrm{V}$ recebiam denominações e símbolos diferentes (às vezes, para a mesma partícula), o físico francês Louis Leprince-Ringuet (1901-2000), da École Polytecnique, em Paris, em 1953141, apresentou um esquema de nomenclatura para as partículas elementares até então conhecidas. Nessa ocasião, denominou de hyperon (do grego iper, que significa "super", "acima" ou "além de") a partícula que apresentava massa maior do que a massa de um núcleon, tais como: $\mathrm{V}^{0, \pm}$ hoje: $\left.\Lambda^{0}\left(\bar{\Lambda}^{0}\right) ; \Sigma^{0, \pm}\left(\bar{\Sigma}^{0, \pm}\right)\right]$. As que apresentavam a massa intermediária entre a massa dos mésons $\left(\pi^{0, \pm}\right)$ e a dos núcleons $(\mathrm{p}, \mathrm{n})$, ele denominou de mésons pesados, os hoje conhecidos káons $\left(\mathrm{K}^{0, \pm}\right)$.

O estudo em detalhes dessas novas partículas só foi possível depois da construção, em 1953, de um acelerador circular (Cosmotron/Bevatron) de $3 \mathrm{BeV}$ [ou $3 \mathrm{GeV}\left(3 \times 10^{9}\right.$ $\mathrm{eV}$ ), sendo GeV o nome hoje mais usado], do Brookhaven National Laboratory (BNL), nos Estados Unidos, e da instalação nesse mesmo laboratório da câmara de bolhas (CB), que havia sido inventada pelo físico norte-americano Donald Arthur Glaser (n.1926; PNF, 1960), em $1952^{142}$, na Universidade de Michigan. É interessante registrar que o primeiro acelerador circular (ciclotron), baseado no fato de que uma partícula de massa m e carga elétrica $\mathrm{q}_{\mathrm{e}}$, ao penetrar com velocidade $\mathrm{v}$ (com módulo v), normalmente às linhas de força de um campo de indução magnética uniforme $\mathrm{B}$ (de intensidade $\mathrm{B}$ ), descreve [devido à Força de Lorentz (1892)] uma circunferência de raio $r=m v /\left(q_{e} B\right)$ e com uma velocidade angular $\omega=q_{e} B / m$,

\footnotetext{
137 Nature, v. 159, p. 694, 1947.

138 Nature, v. 160, p. 453; 486, 1947.

139 Nature, v. 160, p. 855, 1947.

140 Reports Progress in Physics, v. 16, p. 364, 1953.

141 Annual Review of Nuclear Science, v. 3, p. 39, 1953.

142 Physical Review, v. 87, p. 665, 1952.
} 
independente de $\mathrm{v}$ e de $\mathrm{r}$, foi construído pelos físicos norte-americanos Ernest Orlando Lawrence (1901-1958; PNF, 1939) e Milton Stanley Livingston (1905-1986), em 1931143, no Radiation Laboratory, University of California, em Berkeley (RL-UCLA/B). Nesse ciclotron, uma fonte de cargas q (p. ex.: prótons e íons) é colocada no centro de duas caixas na forma de D, uma defronte da outra (com um intervalo entre elas) e normais a B. Este, então, fará a carga q descrever uma circunferência (r). No entanto, quando a mesma descreve metade dessa circunferência, recebe uma energia dada por $\mathrm{q}_{\mathrm{e}} \mathrm{V}$, onde $\mathrm{V}$ é a diferença de potencial devida a um campo elétrico oscilante de frequência $v=\omega /(2 \pi)$, situado entre os dois D e normal ao campo B. Desse modo, toda vez que qe atravessa o intervalo entre os $\mathrm{D}$, ele é acelerado pelo campo elétrico e sua trajetória tem a forma de uma espiral que se inicia na fonte de $\mathrm{q}_{\mathrm{e}}$, em consequência de aceleração múltipla. Depois que qe adquire a energia estabelecida, ele é defletido em um canal e dirigido para o alvo que se deseja bombardear. Com esse equipamento, eles conseguiram produzir prótons (p) de $1 \mathrm{MeV}$.

Muito embora os primeiros ciclotrons funcionassem bem com prótons, eles apresentavam dificuldades em acelerar elétrons, pois a pequena massa (cerca de 1/1.835 da massa do próton) dessas partículas aumentava à medida que sua velocidade crescia, conforme indica a Relatividade Restrita Einsteniana de 1905 ${ }^{144}$; consequentemente, a sua frequência defasava em relação à frequência do campo elétrico oscilante (E). A primeira tentativa para contornar essa dificuldade foi apresentada pelo físico norte-americano Donald William Kerst (19111993), em 1940145, ao construir o primeiro betatron, que acelerava elétrons, oriundos de um núcleo radioativo, com a energia da ordem de $2,5 \mathrm{MeV}$, em uma trajetória circular fixa, uma vez que o aumento relativístico de suas massas era compensado pela variação do B (ver expressão acima) produzido por um indutor. Note que o nome betatron foi dado em virtude de ser o elétron (oriundo de fontes radioativas) conhecido como partícula beta $(\beta)$, segundo já destacamos.

Como o betatron apresentava uma limitação de energia devido ao efeito relativístico do aumento de massa do elétron, os físicos, o russo Vladimir Iosifovich Veksler (1907-1966), em $1944{ }^{146}$ e, independentemente, o norte-americano Edwin Mattison McMillan (1907-1991; PNQ, 1951), em 1945147, propuseram o princípio do sincrociclotron, segundo o qual a frequência do campo elétrico oscilante (E) em um ciclotron era sincronizado com a frequência da partícula acelerada em uma dada órbita. Esse princípio, conhecido como estabilizador de fase, permitiu a construção de aceleradores cada vez mais potentes. O primeiro sincrociclotron, com o diâmetro de 184 polegadas, foi construído em 1947, na Universidade da Califór-

\footnotetext{
143 Physical Review, v. 37, p. 1707; 38, p. 834; 40, p. 19, 1931.

144 BASSALO, J. M. F.; CARUSO, F. Einstein. Livraria da Física, 2013.

145 Physical Review, v. 58, p. 841, 1940.

146 Doklady Akademii Nauk SSSR 43, p. 329; 44, p. 365, 1944.

147 Physical Review, v. 63, p. 143, 1945.
} 
nia, em Berkeley, sob a coordenação de McMillan e Lawrence [com a participação de William Morrison Brobeck (1906-1998) (engenheiro), Kenneth Ross MacKenzie (1912-2002), Robert Serber (1909-1997), D. C. Sewell, K. M. Simpson e R. L. Thornton] e utilizado na aceleração de partículas $\alpha(380 \mathrm{MeV})$.

Apesar dessa inovação no ciclotron, que resultou no sincrociclotron, conforme vimos acima, a aceleração de elétrons com velocidades relativísticas também era um problema para esse novo acelerador. Surgiu então a necessidade de outro dispositivo, no qual o $\mathrm{E}$ fosse mantido constante e o campo magnético (B) é que passasse a variar com o tempo, dispositivo esse que se denominou, mais tarde, de sincrotron. Para o desenvolvimento desse novo acelerador circular e, principalmente, para a redução de seus custos, foi importante o desenvolvimento do princípio conhecido como focagem forte ("strong focusing"). Uma primeira ideia desse princípio foi discutida pelo físico inglês Llewellyn Hilleth Thomas (1903-1992), em 1938148. Mais tarde, em 1949, o engenheiro elétrico grego Nicholas C. Christofilos (19171972) voltou a falar da focagem forte. Note-se que Christofilos, embora nunca tenha publicado essa sua ideia, ele obteve uma patente da mesma em 26 de fevereiro de 1956 (US Patent No. 2.736.799). Registre que, em 1952149, os físicos norte-americanos F. C. Shoemaker, R. J. Britton e B. C. Carlson examinaram a possibilidade da construção de um acelerador de ultraalta energia, usando o referido conceito de focagem forte, cujo objetivo fundamental era o de manter juntas as partículas aceleradas em um ciclotron, durante um enorme número de revoluções e cujas órbitas são contidas em regiões relativamente pequenas, resultando daí sua grande vantagem prática. Por fim, em 1953, os fisicos norte-americanos John Paul Blewett (1910-2000) (de origem canadense) ${ }^{150}$ e, independentemente, Ernest David Courant (n.1920), Linvingston e Hartland S. Snyder (1913-1962) ${ }^{151}$ mostraram como o conceito da focagem forte poderia ser usado em um novo tipo de acelerador: o sincrotron.

O primeiro sincroton usando o princípio da focagem forte, conseguido com uma sucessão de campos magnéticos variáveis e alternados, ou seja, com gradientes de campo magnético alternado, foi construído, em 1954, sob a coordenação do físico norte-americano Robert Rathbun Wilson (1914-2000), na Universidade de Cornell, produzindo elétrons de 1,1 GeV. Por sua vez, o RL-UCLA/B, também na primeira metade da década de 1950, construiu um sincrotron e, com ele, foi possível obter os primeiros antinúcleons. Com efeito, em $1955^{152}$, os físicos norte-americanos Owen Chamberlain (1920-2006; PNF, 1956), Emilio Gino Segrè (1905-1989; PNF, 1959) (de origem italiana), Clyde E. Wiegand (1915-1996) e Thomas John Ypsilantis (1928-2000) usaram-no para produzir o antipróton $(\bar{p})$, estudando a

\footnotetext{
148 Physical Review, v. 54 p. 580; 588, 1938.

149 Physical Review, v. 86, p. 582, 1952.

150 Physical Review, v. 88, p. 197, 1953.

151 Physical Review, v. 88, p. 1190, 1953.

152 Physical Review, v. 100, p. 947, 1955.
} 
colisão de prótons altamente energéticos (da ordem de $\mathrm{BeV}$ ) com átomos de $\mathrm{Cu}$. Note-se que, em 1956, Chamberlain, Segrè, Wiegand e Ypsilantis ${ }^{153}$ previram que o antinêutron $(\bar{n})$ poderia ser produzido na colisão $(\mathrm{p}+\bar{p})$, então, e ainda em $1956^{154}$, os físicos norte-americanos Bruce Cork (1916-1994), Glen R. Lambertson (n.1926) e William A. Wenzel e o italiano Oreste Piccioni (1915-2002), trabalhando no RL-UCLA/B, confirmaram essa previsão fazendo passar um feixe de $\bar{p}$ através da matéria. Note-se que, usando ainda a focagem forte, foi construído, em 1959, no Conseil Européen pour la Recherche Nucléaire (CERN) (criado em 1952 e iniciado seu funcionamento em 1954), o sincrotron de próton [Proton Synchrotron (PS)] de $28 \mathrm{GeV}$ e, em 1960, o acelerador de prótons ou Alternating Gradient Synchrotron (AGS), no Brookhaven National Laboratory (BNL), de $33 \mathrm{GeV}$.

Ainda na década de 1950, a atualização dos sincrotrons permitiu produzir outras partículas (com suas antipartículas) mais pesadas que os núcleons [por exemplo: $\Xi^{0,-}\left(\bar{\Xi}^{0,-}\right)$, $\Delta^{++,+, 0,-}$ e que receberam o nome de bárions (do grego barys, que significa "pesado") e cunhado pelo físico e historiador da ciência, o holandês-norte-americano Abraham Pais (19182000), em 1954155. Note-se que, em 1946, os então conhecidos elétron (e $\left.{ }^{-}\right)$e pósitron $\left(\mathrm{e}^{+}\right)$[e seus previstos (neutrinos/antineutrinos: $\left.v_{\mathrm{e}}, \bar{v}_{e}\right)$ ] e múons $\left(\mu^{-,+}\right)$receberam de Möller e Pais ${ }^{156}$, a denominação de léptons (do grego leptos, que significa "fino" ou "pequeno") e, independentemente, pelo físico belga Léon Rosenfeld (1904-1974), em seu livro Nuclear Forces ${ }^{157}$, publicado em 1948. Note-se que, hoje, os léptons (e seus respectivos antiléptons) são em número de seis cada $\left[\left(\mathrm{e}^{-,+} ; v_{\mathrm{e}}, \bar{v}_{e}\right) ;\left(\mu^{-,+} ; v_{\mu}, \bar{v}_{\mu}\right) ;\left(\tau^{-,+} ; v_{\tau}, \bar{v}_{\tau}\right)\right]$.

Por sua vez, a descoberta, também na década de 1950 e no começo da década de 1960 , de novas partículas "estranhas" com uma vida média extremamente pequena $\left(\approx 10^{-23} \mathrm{~s}\right)$ [então denominadas ressonâncias": "mesônicas" $\left(\mathrm{K}^{*, 0,+} ; \overline{\mathrm{K}}^{* 0} ; \mathrm{K}^{*+}\right)$ e "bariônicas" ( $\Sigma^{* 0+,-}$; $\left.\Delta^{++,-}\right)$], assim como os mésons-rô $\left(\rho^{0,+,-}\right)$ levou, em 1962158, o físico russo Lev Borisovich Okun (n.1929) a denominar de hádrons (do grego adros, que significa "grosso e volumoso"), partículas constituídas de bárions (núcleons e hyperons) e de mésons. Registre-se que os hádrons e léptons são as partículas que constituem a matéria, denominadas por isso de portadoras da matéria (e que podem ser férmions ou bósons).

Na década de 1960, foram desenvolvidos modelos matemáticos para entender as partículas hadrônicas, tendo como base a Teoria de Grupos, em particular o Grupo Unitário Especial (“Special Unitary Group”) de grau n: SU(n), caracterizado por ter seu determinante

\footnotetext{
153 Nature, v. 177, p. 11, 1956.

154 Physical Review, v. 104, p. 1193, 1956.

155 Proceedings of the Internacional Conference on Theoretical Physics 1954, Kyoto, p. 157, 1954.

156 ABRAHAM PAIS. A Tale of Two Continents: A Physicist's Life in a Turbulent World. Princeton University Press, 1997.

157 Interscience Publishers, New York.

158 Proceedings of the International Conference on High Energy Physics 1962, CERN, p. 845, 1962.
} 
unitário. Assim, em 1964159, o físico norte-americano Murray Gell-Mann (n.1929; PNF, 1969) usou o SU(3) para desenvolver o modelo de quarks, segundo o qual os hádrons eram constituídos de novas partículas, os quarks, da seguinte maneira: bárions constituídos de três quarks (q) e os mésons de pares de quark-antiquark (q, $\bar{q}$ ). Ainda em 1964 (CERN Preprint 8182/Th 401; 8419/Th 412), o físico russo-norte-americano George Zweig (n.1937) desenvolveu um modelo análogo a esse de Gell-Mann, no entanto, deu o nome de aces a essas novas partículas. Observe-se que tais partículas se apresentam em três sabores ("flavours"): up (u), down (d) e strange (s) e seus respectivos antiquarks: $\bar{u}, \bar{d}, \bar{s}$. Tais partículas são fermiônicas $($ spin $=1 / 2)$ e têm cargas fracionárias dadas por: $+2 \mathrm{e} / 3,-\mathrm{e} / 3,-\mathrm{e} / 3$ (para os quarks $) ;-2$ $\mathrm{e} / 3,+\mathrm{e} / 3,+\mathrm{e} / 3$, (para os antiquarks) onde "e" representa a carga do elétron. Registre-se que Gell-Mann deu o nome de quark para homenagear o escritor irlandês James Joyce (18821941), uma vez que em uma das estrofes do livro Finnegan's Wake, escrito por Joyce em 1939, lê-se: - Three quarks for Master Mark. Registre-se ainda que como decorrência do MQ, foram previstos (p.) e posteriormente descobertos (d.) mais três quarks e respectivos antiquarks: charmoso (charm) [c, $\bar{c}( \pm 2 \mathrm{e} / 3$; p.1964; d.1974)]; bonito (bottom) $[\mathrm{b}, \bar{b}$ ( $\mp \mathrm{e} / 3$; p.1974; d.1977)]; e top [t, $\bar{t}$ ( \pm 2 e/3; p.1974; d.1995)].

A primeira grande dificuldade do MQ surgiu quando foi observado que três hádrons eram formados de três quarks iguais, a saber: $\Delta^{++}$(uuu) e $\Delta^{-}$(ddd) (d.1953) e $\Omega^{-}$(sss) (d.1964). Porém, como os quarks são férmions, essas três partículas violariam o Princípio da Exclusão de Pauli (PEP) (não se pode juntar mais de dois férmions com as mesmas características físicas), formulado em 1925, como vimos acima. Assim, para contornar essa dificuldade, o físico norte-americano Oscar Wallace Greenberg (n.1932), em 16 de novembro de $1964{ }^{160}$, propôs que cada quark fosse caracterizado por uma das três cores primárias do espectro luminoso: vermelho ("red" - r), azul ("blue" - b) e verde ("green" - g), que representavam um novo número quântico: cor. Por sua vez, os antiquarks seriam caracterizados pelas cores complementares desse mesmo espectro: ciano ("cyan" - c), amarelo ("yellow" - y) e magenta ("magenta" - m). Assim, teríamos, por exemplo: $\Delta^{++}\left(\mathrm{u}_{\mathrm{r}} \mathrm{u}_{\mathrm{b}} \mathrm{u}_{\mathrm{g}}\right), \Delta^{-}\left(\mathrm{d}_{\mathrm{r}} \mathrm{d}_{\mathrm{b}} \mathrm{d}_{\mathrm{g}}\right)$ e $\Omega\left(\mathrm{s}_{\mathrm{r}} \mathrm{SbSg}_{\mathrm{g}}\right)$. Note-se que essa proposta de Greenberg foi confirmada pelos físicos, o koreano Moo-Young Han (n.1934) e o japonês Yoichiro Nambu (1921-2015; PNF, 2008), em $1965^{161}$.

Com a inclusão de mais um número quântico na família dos hádrons, havia necessidade de se desenvolver uma Teoria Quântica de Campos (TQC) para os mesmos. Com efeito, em analogia com a QED [segundo a qual a força (interação) eletromagnética (fem) entre cargas elétricas decorre da troca de fótons $(\gamma)$ entre as mesmas, como vimos acima], em 1973, foram realizados trabalhos independentes pelos físicos norte-americanos David Jonathan

\footnotetext{
159 Physics Letters, v. 8, p. 214, 1964.

160 Physical Review Letters, v. 13, p. 598, 1964.

161 Physical Review B139, p. 1006, 1965.
} 
Gross (n.1941; PNF, 2004) e Frank Anthony Wilczek (n.1951; PNF, 2004) ${ }^{162}$, e Hugh David Politzer (n.1949; PNF, 2004)163, trabalhos esses nos quais formularam a Cromodinâmica Quântica (QCD: Quantum Chromodynamics), segundo a qual a força forte (fo) entre os quarks seria consequência da troca entre si das partículas glúons (g) (do inglês glue, que significa "cola) que são bosônicas (spin 1) e não-massivas. Tais partículas seriam responsáveis pela cor do quark, e este novo número quântico (c) representaria na QCD o mesmo papel que a carga elétrica (e) representa na QED.

Ainda segundo a QCD, para que os quarks (que são férmions, já registrado) se mantenham sempre juntos sem violar o PEP, deverão trocar glúons entre si a fim de mudarem de cor. Por exemplo, um quark vermelho para se transformar em quark azul, emite um glúon vermelho-amarelo, pois o amarelo é o antiazul. É oportuno registrar que enquanto na força forte há troca de cor entre os quarks, na força fraca há troca de sabor entre eles. Desse modo, por exemplo, no caso do decaimento $\beta$ (em notação quarkônica): $\mathrm{n}$ (udd) $\rightarrow \mathrm{p}$ (uud) $+\mathrm{e}^{-}+\bar{v}_{e}$, pois: $\mathrm{d} \rightarrow \mathrm{u}+\mathrm{W}^{-}$e $\mathrm{W}^{-} \rightarrow \mathrm{e}^{-}+\bar{v}_{e}$. Note-se que o físico sueco Oskar Benjamin Klein (18941977), em $1938^{164}$, propôs que aquele decaimento seria mediado por bósons vetoriais ( $\operatorname{spin}=$ 1) massivos e carregados, aos quais denominou de $\omega$ (de weak, fraco em inglês) e hoje $W^{ \pm}$.

Ainda sobre as PE, é interessante registrar que os físicos, o norte-americano Steven Weinberg (n.1933; PNF, 1979)165 e o paquistanês Abdus Salam (1926-1996; PNF, 1979)166 formularam a conhecida Teoria Eletrofraca ou Teoria de Salam-Weinberg (TEF/TSW), que unifica as forças eletromagnética (fem) e fraca (fr). Segundo essa teoria, a "força eletrofraca" é mediada por quatro (4) quanta: o fóton $(\gamma)$ mediador da fem e os bósons vetoriais, sendo: os $\mathrm{W}^{ \pm}$mediadores das reações nucleares envolvendo correntes carregadas fracas e o $\mathrm{Z}^{0}$ (notação sugerida por Weinberg) nas correntes neutras fracas. Convém registrar que a TEF/TSW apresentava uma grande dificuldade, pois ela não era renormalizável, ou seja, apareciam divergências (infinitos) nos cálculos envolvendo os 4 quanta, característicos daquela teoria. A referida dificuldade foi resolvida, em 1972 ${ }^{167}$, pelos físicos holandeses Gerardus 't Hooft (n.1946; PNF, 1999) e Martinus Justinus Godefridus Veltman (n.1931; PNF, 1999) e, desse modo, a TEF/TSW, junto com a QED e a QCD, completaram o MPPE.

Para concluir este pequeno resumo histórico do MPPE, é oportuno registrar a estrutura quarkônica de alguns hádrons (bárions e mésons). Vejamos primeiro os bárions: próton [p $=$ uud (d. 1919)]; nêutron $\left[\mathrm{n}=\mathrm{udd}\right.$ (d. 1932)]); lambda charmoso-mais $\left[\Lambda_{c}^{+}=\mathrm{udc}\right.$ (d. 1975)]; xi charmoso-mais $\left[\Xi_{c}^{+}=\right.$usc (d. 1983)]; xi charmoso-charmoso-mais-mais $\left[\Xi_{c c}^{++}=\right.$ucc $(\mathrm{d}$.

\footnotetext{
162 Physical Review Letters, v. 30, p. 1343, 1973.

163 Physical Review Letters, v. 30, p. 1346, 1973.

${ }^{164}$ Journal de Physique et le Radium, v. 9, p. 1, 1938.

165 Physical Review Letters, v. 19, p. 1264, 1967.

166 Proceedings of the Eighth Nobel Symposium, p. 367, 1969.

167 Nuclear Physics B44, p. 189; B50, p. 189, 1972.
} 
2017)]; lambda bonito-zero $\left[\Lambda_{b}^{0}=\mathrm{udb}(\mathrm{d} .1991)\right]$; sigma charmoso-zero;mais-mais $\left[\Sigma_{c}^{0}=\mathrm{ddc}\right.$; $\Sigma_{c}^{++}=$uuc (d.1996)]; xi bonito-menos $\left[\Xi_{b}^{-}=\mathrm{dsb}\right.$ (d.2007)]; ômega bonito $\left(\Omega_{b}^{-}=\operatorname{ssb}(\mathrm{d} .2008)\right]$. E agora, os mésons: píon-menos $\left[\pi^{-}=\bar{u} \mathrm{~d}\right.$ (d.1947)]; píon-mais $\left[\pi^{+}=\mathrm{u} \bar{d}\right.$ (d.1947)]; píon-zero $\left[\pi^{0}=\mathrm{d} \bar{d}\right.$ (d.1950); káon-menos $\left[\mathrm{K}^{-}=\bar{u} \mathrm{~s}\right.$ (d.1949)]; káon-mais $\left[\mathrm{K}^{+}=\mathrm{u} \bar{s}\right.$ (d.1949)]; káon-zero $\left[\mathrm{K}^{0}=\mathrm{d} \bar{s}(\mathrm{~d} .1951)\right]$; antikáon-zero $\left[\bar{K}^{0}=\bar{d} \mathrm{~s}(\mathrm{~d} .1951)\right]$; eta-zero $\left[\eta^{0}=\mathrm{u} \bar{u}\right.$ (d.1961)]; eta linha-zero $\left[\eta^{\prime 0}=\mathrm{s} \bar{s}\right.$ (d.1964)]; rho-menos [ $\left.\rho^{-}=\bar{u} \mathrm{~d}(\mathrm{~d} .1961)\right]$; rho-mais $\left[\rho^{+}=\mathrm{u} \bar{d}\right.$ (d.1961)]; rho-zero $\left[\rho^{0}=\mathrm{d} \bar{d}\right.$ (d.1961)]; káon-estrela-zero $\left[\mathrm{K}^{* 0}=\mathrm{d} \bar{s}\right.$ (d.1961)]; káon-estrela-mais $\left[\mathrm{K}^{*+}\right.$ $=\mathrm{u} \bar{s}(\mathrm{~d} .1961)]$; káon-estrela-menos $\left[\mathrm{K}^{*-}=\bar{u} \mathrm{~s}(\mathrm{~d} .1961)\right]$; antikáon-estrela-zero $\left[\bar{K}^{* 0}=\bar{d} \mathrm{~s}\right.$ (d.1961)]; ômega-zero $\left[\omega^{0}=\mathrm{u} \bar{u}(\mathrm{~d} .1961)\right]$; phi-zero $\left[\phi^{0}=\mathrm{s} \bar{s}(\mathrm{~d} .1963)\right]$; psi/jota $[\psi / J=\mathrm{c} \bar{c}$ (d.1974)]; D-zero $\left[\mathrm{D}^{0}=\bar{u} \mathrm{c}(\mathrm{d} .1975)\right]$; D-mais $\left[\mathrm{D}^{+}=\bar{d} \mathrm{c}\right.$ (d.1976)]; upsilon-zero $\left[\Upsilon^{0}=\mathrm{b} \bar{b}\right.$ (d.1977)]; e mésonB-zero $\left[\mathrm{B}^{0}=\bar{b} \mathrm{~d}(\mathrm{~d} .1987)\right]$.

Como observação final sobre o MPPE, é interessante registrar que ele apresenta dois resultados importantes (e motivo de questionamento): os léptons não possuem estrutura quarkônica, e os neutrinos não têm massa, mas estranhamente oscilam.

\section{Modelo Padrão do Big Bang}

Visto o pequeno histórico do MPPE, tratemos do MPBB. Em, 1915 ${ }^{168}$, Einstein formulou a Teoria da Relatividade Geral (TRG) traduzida pela Equação de Einstein (EE): $\mathrm{R}_{\mu \nu}-$ $1 / 2 \mathrm{~g}_{\mu \nu} \mathrm{R} \equiv \mathrm{G}_{\mu \nu}=\mathrm{K}_{\mu \nu}$; onde $\mathrm{g}_{\mu v}\left(\mathrm{~g}^{\mu \nu}\right)$ é o tensor métrico riemanniano; $\mathrm{R}_{\mu \nu}$ é o tensor geométrico de Ricci; $\mathrm{G}_{\mu \nu}$ é o tensor de Einstein; $\mathrm{T}_{\mu \nu}$ é o tensor energia-matéria; $\mathrm{R}=\mathrm{g}^{\mu \nu} \mathrm{R}_{\mu \nu}$ é a curvatura escalar de Ricci; $\mathrm{K}=8 \pi \mathrm{G} / \mathrm{c}^{4}$ é a constante de gravitação de Einstein; $\mathrm{G}$ é a constante de gravitação de Newton-Cavendish; c é a velocidade da luz no vácuo; e $\mu, v=0,1,2$, 3. Note-se que a TRG explica a força (interação) gravitacional (fg), que é uma das quatro forças da natureza, sendo as outras três: eletromagnética (fem), fraca (fr) e forte (fo), cujos significados foram vistos no resumo histórico do MPPE apresentado anteriormente.

Logo depois, em 1916 169 , o astrônomo alemão Karl Schwarszchild (1873-1916) encontrou uma solução rigorosa para a EE, ao considerar uma carga puntiforme de massa m colocada em um campo gravitacional (cg) isotrópico e estático. Essa solução ficou mundialmente conhecida como a Métrica de Schwarszchild e que, no entanto, apresentava a singularidade infinita $(\infty):(1-2 \mathrm{mG} / \mathrm{r})^{-1}$, e que ocorre quando $\mathrm{r}=2 \mathrm{~m} \mathrm{G}$, hoje conhecido como o raio $\left(\mathrm{r}_{\mathrm{BN}}\right)$ de um buraco negro ("black hole") $(\mathrm{BN})$.

Em 1917170, Einstein encontrou uma solução para a sua equação que era uma solução dinâmica. Note-se que, por essa época, não havia nenhuma evidência experimental sobre a dinâmica do Universo, isto é, se o seu raio dependia do tempo. Então, para contornar essa

\footnotetext{
168 Sitzungsberichte Preussische Akademie der Wissenchaften, v. 2, p. 844, 1915.

${ }^{169}$ Sitzungsberichte Preussische Akademie der Wissenchaften, v. 2, p. 189; 424, 1016.

170 Sitzungsberichte Preussische Akademie der Wissenchaften, v. 1, p. 142, 1917.
} 
dificuldade, ele formulou a hipótese de que as forças entre as galáxias eram independentes de suas massas e que variavam na razão direta da distância entre elas, isto é, havia uma "repulsão cósmica", além, é claro, da "atração gravitacional Newtoniana". Matematicamente, essa hipótese significava acrescentar um termo ao primeiro membro de sua equação - o famoso termo cosmológico ou termo de repulsão cósmica $\left(\Lambda \mathrm{g}_{\mu \nu}\right)$. Desse modo, Einstein postulou que o Universo era estático e, usando sua equação, demonstrou ser o mesmo finito e de curvatura riemanniana positiva ou esférica. Em virtude disso, o seu modelo cosmológico ficou conhecido como o Universo Cilíndrico de Einstein, em que o espaço é curvo, porém o tempo é retilíneo. Note-se que, em 1922171, o matemático russo Aleksandr Friedmann (1888-1925) resolveu a $\mathrm{EE} \operatorname{com} \Lambda=0$ e, ao assumir a hipótese de que a matéria homogênea do Universo se distribuía isotropicamente no espaço, encontrou duas soluções não estáticas: em uma delas o Universo se expandia com o tempo e, na outra, se contraía. Note-se que esse Universo, na linguagem de hoje, era um estado de vácuo quântico com densidade de energia infinita.

A possibilidade teórica de um Universo em Expansão prevista por Friedmann começou a se tornar realidade devido aos trabalhos realizados pelo astrônomo norte-americano Edwin Powell Hubble (1889-1953). Com efeito, em dezembro de 1924, trabalhando com o novo telescópio Hooker do Observatório de Monte Wilson, Hubble estava examinando uma fotografia da nebulosa (galáxia) de Andrômeda (M31) [M, do catálogo preparado pelo astrônomo francês Charles Messier (1730-1817), em 1771]. Nesse exame, encontrou uma estrela do mesmo tipo existente em nossa nebulosa (galáxia): a Via Láctea. Continuando a estudar as nebulosas fora de nossa Galáxia, chegou à seguinte conclusão: - As galáxias são distribuídas no espaço de modo homogêneo e isotrópico.

Assim, pela primeira vez, a uniformidade do Universo não era colocada a priori, ela provinha de uma observação. Essas observações de Hubble foram publicadas em $1925^{172}$ e em 1926173. Logo depois, em 1927174, o astrônomo belga, o Abade Georges-Henri Edouard Lemaître (1894-1966) confirmou teoricamente o modelo dinâmico do Universo e, nessa ocasião, afirmou que o Universo teria começado a partir da explosão de um átomo primordial ou ovo cósmico. É oportuno registrar que, em 1929175, Hubble fez outra grande descoberta ao observar cerca de 18 galáxias próximas de nossa Galáxia e percebeu que havia no espectro das mesmas um deslocamento para o vermelho (red shift). Interpretado esse deslocamento como devido ao Efeito Doppler (1842)-Fizeau (1848) \{aumento do comprimento de onda $(\lambda)$ [correspondente à diminuição de sua frequência $(v)$, pois $\mathrm{c}=\lambda v]$ de uma radiação eletromagnética ao se aproximar da fonte receptora \}, o mesmo significava uma "fuga" das galáxias, em rela-

171 Zeitschrift für Physik, v. 10, p. 377, 1922.

172 Astrophysical Journal, v. 62, p. 409, 1925.

173 Astrophysical Journal, v. 63, p. 236; 64, p. 321, 1926.

${ }^{174}$ Annales de la Societé Scientifique de Bruxelles, v. 47A, p. 49, 1927.

175 Proceedings of the National Academy of Sciences 15, p. 169, 1929. 
ção ao observador. Ao calcular a distância entre as várias galáxias, concluiu que (logo conhecida como Lei de Hubble): - As galáxias se afastam uma das outras com uma velocidade (V) proporcional à distância $(\mathrm{D})$ que as separam. A proporcionalidade $\left(\mathrm{H}_{0}\right)$ entre $\mathrm{V}$ e $\mathrm{H}$, traduzida pela expressão $\mathrm{V}=\mathrm{H}_{0} \mathrm{D}$, foi estimada por Hubble, ainda nessa ocasião, no valor de: $\mathrm{H}_{0} \approx$ $0,5 \times 10^{-9}$ anos, significando dizer que a explosão do "ovo cósmico lemaîtriano" acontecera há cerca de 1 bilhão de anos (ba) (hoje: $\approx 13,7$ ba). Como, em 1934 ${ }^{176}$, Hubble e o astrônomo norte-americano Milton La Salle Humason (1891-1972) fizeram um novo cálculo para $\mathrm{H}_{0}$, aquela lei passou então a ser conhecida como Lei de Humason-Hubble, usada até hoje para calcular a idade do Universo e que corresponde ao inverso de $\mathrm{H}_{0}$.

O Modelo Cosmológico de Friedmann-Lemaître foi estudado, em $1948^{177}$, pelos físicos norte-americanos Ralph Asher Alpher (1921-2007), Bethe (de origem alemã) e Gamow (de origem russa), ocasião em que formularam o famoso modelo cosmológico $\alpha \beta \gamma$ (Alpher, Bethe, Gamow), no qual o "ovo cósmico lemaîtriano" formado de nêutrons (n), no instante do Big Bang (BB), se desintegrou em prótons (p) e elétrons ( $\mathrm{e}^{-}$) [ver o decaimento $\beta: \mathrm{n} \rightarrow \mathrm{p}+\mathrm{e}^{-}$ $\left.+v\left(\bar{v}_{e}\right)\right]$. Ao serem formados esses $\mathrm{p}$, alguns colidiram com $\mathrm{n}$ que ainda persistiam e, gradualmente, iam formando os núcleos mais pesados da Tabela Periódica dos Elementos, num processo que ficou conhecido como nucleossíntese e, a temperatura correspondente a essa explosão, conhecida como radiação cósmica de fundo de micro-onda (RCFM) [“Cosmic Microwave Background" (CMB)], foi determinada, também em 1948 ${ }^{178}$, por Alpher e o físico norte-americano Robert C. Herman (1922-1997), também colaborador de Gamow, que encontraram para a RCFM [usando a Lei de Stefan-Boltzmann, desenvolvida pelos físicos austríacos Josef Stefan (1835-1893), em 1879179 e Ludwig Boltzmann (1844-1906), em 1884180] um valor de aproximadamente $5 \mathrm{~K}$. É interessante destacar que a RCFM foi detectada com o valor de $(3.5 \pm 1) \mathrm{K}$, em 1965, em trabalhos independentes dos norte-americanos, os astrofísicos Robert Henry Dicke (1916-1997), Phillip James Edwin Peebles (n.1935), Peter Guy Roll e David Todd Wilkinson (1935-2002)181 e os radio-astrônomos Arno Allan Penzias (n.1933; PNF, 1978) (de origem alemã) e Woodrow Wilson (n.1936; PNF, 1978) ${ }^{182}$. Note-se que Penzias e Wilson, em certo dia de maio de 1934, haviam usado uma antena de 20 pés que havia sido construída pela Bell Telephone Laboratories em Holmdel, New Jersey, nos Estados Unidos e mediram por intermédio dos satélites Echo e Telstar, micro-ondas galácticas de 7,35 $\mathrm{cm}$, em latitudes fora do plano da Via Láctea e, desse modo, descobriram que tais micro-

\footnotetext{
176 Astrophysical Journal, v. 74, p. 43, 1934.

177 Physical Review, v. 73, p. 803, 1948.

178 Physical Review, v. 74, p. 1198, 1948.

${ }^{179}$ Sitzungsberichte der Kaiserlichen Akademie der Wissenchaften zu Wien, v. 79, p. 391, 1879.

180 Annalen der Physik, v. 22, p. 31; 291, 1884.

181 Astrophysical Jornal, v. 142, p. 414, 1965.

182 Astrophysical Jornal, v. 142, p. 419, 1965.
} 
ondas eram independentes da direção apontada pela antena, isto é, eram isotrópicas. É oportuno registrar que o termo Big Bang (BB) foi cunhado, pejorativamente, pelo astrofísico inglês Sir Fred Hoyle (1915-2001), no último programa de rádio de uma série intitulada The Nature of Things que apresentou na British Broadcasting Corporation (BBC), em 1950, uma vez que, também em 1948, e em trabalhos independentes, Hoyle 183 e os astrofísicos, o austroinglês Sir Hermann Bondi (1919-2005) e o austro-norte-americano Thomas Gold (1920$2004)^{184}$, haviam proposto o então conhecido modelo cosmológico estacionário de BondiGold-Hoyle (BGH).

Muito embora a detecção da RCFM, em 1965, tenha dado bastante crédito ao MPBB, este começou a ser contestado nas décadas de 1960 e 1970, em virtude de sua dificuldade em explicar quatro grandes problemas (puzzles). O primeiro deles, conhecido como problema do horizonte (horizon puzzle), refere-se à homogeneidade e isotropia do Universo; o segundo, conhecido como problema da planura (flatness puzzle), diz respeito à densidade $\Omega$ de massa do Universo, cujo valor, de acordo com o MPBB, é dado por: $\Omega-1$ proporcional a $\mathrm{t}^{2(1-\mathrm{n})}$, com $\mathrm{n}<1$. Assim, se $\Omega<1$, a densidade de massa é insuficiente para deter a expansão, e o Universo continuará a expandir-se para sempre; se $\Omega>1$, a expansão acabará, e o Universo presumivelmente colapsará em outra "bola de fogo" (big crunch), significando que ele é $f e$ chado; por fim, se $\Omega=1$, então a expansão seguirá para sempre, e sempre diminuindo, mas sem chegar nunca a parar totalmente, indicando que o Universo é plano. O terceiro dos problemas enfrentados pelo MPBB relaciona-se com as inhomogeneidades (inhomogeneity puzzle) do Universo observável, composto de galáxias, aglomerados de galáxias e superaglomerados de galáxias, uma vez que, por aquela teoria, esse espectro de não-uniformidade deve ser considerado ad hoc no MPBB, como parte de suas condições iniciais. Por fim, o quarto problema tem relação com a produção de monopolos magnéticos (MM) na ocasião do início do Universo, daí esse problema ser conhecido como o problema dos monopolos (monopole puzzle). Destaque-se que tais partículas, previstas pelo físico inglês Paul Adrien Maurice Dirac (1902-1984; PNF, 1933), em 1931'185, ainda não foram observadas.

Para contornar os problemas apresentados pelo MPBB descritos acima, acrescido da presença incômoda de $\Lambda$, os físicos, o russo Aleksandr A. Starobinsky (n.1950), em 1979186, e o norte-americano Alan Harvey Guth (n.1947), em 1981 ${ }^{187}$, formularam o Modelo Cosmológico Inflacionário (MCI), segundo o qual o Universo teria começado também com um $\mathrm{BB}$, ocorrido entre $(15$ e 20$) \times 10^{9}$ anos atrás. No instante do BB $(\mathrm{t}=0 \mathrm{~s})$, houve criação de matéria e de antimatéria [constituídas de partículas de carga elétrica $\left(\mathrm{e}^{ \pm, 0}\right)$ ], em quantidades

\footnotetext{
183 Monthly Notices of the Royal Astronomical Society, v. 108, p. 372, 1948.

${ }^{184}$ Monthly Notices of the Royal Astronomical Society, v. 108, p. 252, 1948.

185 Proceedings of the Royal Society of London A133, p. 60, 1931.

186 Pis'ma v Zhurnal Eksperimental'noi I Teoreticheskoi Fiziki, v. 30, p. 719, 1979.

${ }^{187}$ Physical Review D23, p. 347, 1981.
} 
iguais, assim como as quatro forças da natureza (fg, fem, fr, fo) também foram "criadas", mas estavam acopladas. "supostamente" regida por uma única lei física (envolvendo certas simetrias), traduzida pelo hoje famoso Teorema CPT, que é o princípio segundo o qual os sistemas físicos são invariantes para as transformações (invariância "gauge") que envolvem, concomitantemente, as operações de inversão da carga $(\mathrm{C}$ : troca de e por - e), inversão de paridade $(\mathrm{P}$ : troca de $\mathrm{r}$ por - r) e inversão temporal (T: troca de $\mathrm{t}$ por $-\mathrm{t}$ ).

É oportuno registrar que, em 1952188, Wick e os físicos norte-americanos Arthur Strong Wightman (n.1922) e Eugene Paul Wigner (1902-1995; PNF, 1963) (de origem húngara) propuseram que reações entre as partículas/antipartículas obedeciam à simetria (invariância) carga-paridade (CP). Contudo, em 1964, experiências independentes realizadas pelos físicos, os norte-americanos James Henry Christenson (1935-2015), James Watson Cronin (1931-2016; PNF, 1980), Val Logsdon Fitch (1923-2015; PNF, 1980) e o francês René Turlay (1932-2002)189, e A. Abashian, R. J. Abrams, D. W. Carpenter, G. P. Fisher, Bernard ("Ben”) Marie Karel Nefkens (1934-2014) e James H. Smith ${ }^{190}$, observaram a violação da CP do sistema $\mathrm{K}^{0}-\bar{K}^{0}$.

Voltemos ao MPBB. À medida que o tempo fluía, constituiu-se a conhecida Era de Planck (EP), como veremos a seguir. Assim, ocorrido $10^{-43}$ seg $\left\{\right.$ Tempo de Planck: $\mathrm{t}_{\mathrm{P}}=[\mathrm{G}$ $\left.\left.\mathrm{h} /\left(2 \pi \mathrm{c}^{5}\right)\right]^{1 / 2}\right\}$, o Universo apresentava um comprimento de $\approx 10^{-33} \mathrm{~cm}$ \{Comprimento de Planck: $\left.\ell_{\mathrm{P}}=\left[\mathrm{G} \mathrm{h} /\left(2 \pi \mathrm{c}^{3}\right)\right]^{1 / 2}\right\}$; uma massa (homogênea e isotrópica) de $\approx 10^{-5} \mathrm{~g}$ \{Massa de Planck: $\left.\mathrm{m}_{\mathrm{P}}=[\mathrm{c} \mathrm{h} /(2 \pi \mathrm{G})]^{1 / 2}\right\} ;$ uma energia de $\approx 10^{19} \mathrm{GeV}$ \{Energia de Planck: $\mathrm{E}_{\mathrm{P}}=\mathrm{m}_{\mathrm{P}} \mathrm{c}^{2}=$ $\left.\left[\mathrm{c}^{5} \mathrm{~h} /(2 \pi \mathrm{G})\right]^{1 / 2}\right\}$; e uma temperatura de $\approx 10^{32}$ graus Kelvin $(\mathrm{K})$ \{Temperatura de Planck: $\mathrm{T}_{\mathrm{P}}$ $\left.=\mathrm{E}_{\mathrm{P}} / \mathrm{k}=\left[\mathrm{c}^{5} \mathrm{~h} /\left(2 \pi \mathrm{G} \mathrm{k}^{2}\right)\right]^{1 / 2}\right\}$. Observe-se que, decorrido $10^{-35} \mathrm{~s}$ contado a partir do BB, o Universo sofreu um período de expansão muito acelerada, isto é, uma inflação, durante o qual o o Universo aumentou cerca de $10^{50}$ vezes e sua temperatura baixou para $\approx 10^{27} \mathrm{~K}$.

Continuemos com o histórico do MPBB. O MCI apresentou explicações para três dos problemas ("puzzles") do BB. Com efeito, como o Universo teria sido muito maior no fim do período inflacionário do que o previsto pelo MPBB, o espaço seria muito mais achatado, o que explicaria o problema da planura. Por outro lado, por ser mais chato e liso o Universo na época da transição de fase entre a época inflacionária e o ritmo linear do Universo de hoje, produziram-se muito menos MM, o que resolveria o problema dos MM. Quanto ao problema do horizonte, o MCI apresentou a seguinte explicação: logo depois que o Universo se iniciou (no $t_{\mathrm{P}}$ ), a fg desaclopou-se das outras três forças (fem, fr, fo) e ele sofreu um tipo de superresfriamento, produzindo um "congelamento" destas forças, por intermédio de um mecanismo denominado de transição de rolamento lento ("slow rolleyer transition"). Desse modo, quan-

\footnotetext{
188 Physical Review, v. 88, p. 101, 1952.

189 Physical Review Letters, v. 13, p. 138, 1964.

190 Physical Review Letters, v. 13, p. 243, 1964.
} 
do ocorreu a fase inflacionária $\left(10^{-35}-10^{-33}\right) \mathrm{s}$, quaisquer irregularidades do Universo foram simplesmente aplainadas, daí a sua homogeneidade e isotropia atuais.

Muito embora o MCI tivesse contornado as dificuldades do MPBB, conforme vimos acima, aquele modelo apresentava outro problema, pois, se a fase de transição entre o Universo "super-resfriado" e o estado de expansão linear atual ocorresse subitamente, haveria a formação de "bolhas" (como ocorre no surgimento de cristais de gelo em qualquer água superfria) que se expandiriam gradualmente e se juntariam umas às outras, até a situação de expansão linear do Universo que perdura até hoje. Contudo, mesmo que as "bolhas" crescessem à velocidade da luz, estariam se afastando umas das outras e, portanto, nunca se juntariam. Essa dificuldade foi resolvida, em 1982, em trabalhos independentes realizados pelos físicos, o russo Andrei Dimitrievich Linde (n.1948) ${ }^{191}$, e os norte-americanos Andréas Albrecht e Paul J. Steinhardt (n.1952) ${ }^{192}$, ao formularem o Novo Modelo Cosmológico Inflacionário (NMCI), segundo o qual o fato de as "bolhas" não se juntarem poderia ser evitado se elas fossem tão grandes que nossa região do Universo estivesse toda contida numa única bolha. No entanto, para que isso ocorresse, a "quebra de simetria" deveria acontecer muito lentamente dentro da "bolha", o que é perfeitamente possível de acordo com a Teoria de Grande Unificação (TGU) [unificação das fem, fr e fo e conhecida como força eletronuclear (fen)], por intermédio de um mecanismo denominado de transição de rolamento lento ("slow rolleyer transition"), segundo a qual o próton é uma partícula instável (vida média $\approx 10^{31}$ anos) e tendo o MM como um de seus produtos de decaimento. Note-se que a TGU se deve aos trabalhos dos físicos, o paquistanês Salam e o indiano Jogesh C. Pati (n.1937), em 1972193, e aos norte-americanos Sheldon Lee Glashow (n.1932; PNF, 1979) e Howard Mason Georgi (n.1947), em 1974194.

Como novos problemas foram encontrados nesse NMCI como, por exemplo, o fato de que a "bolha" única deveria ser maior do que o Universo à época, conforme foi mostrado pelo astrofísico inglês Stephen William Hawking (n.1942) e seus colaboradores Ian G. Moss e John M. Stewart (1943-2016), em 1982195, e que as flutuações quânticas iniciais deveriam crescer mais do que o esperado, indicando um tempo de rolamento muito mais lento, o próprio Linde, em 1983196, apresentou o Modelo Cosmológico Inflacionário Caótico (MCIC), no qual não há transição de fase ou super-resfriamento. A ideia central desse modelo consiste em supor uma distribuição inicial caótica de um campo $\phi$ de bósons de Higgs, cujas flutuações quânticas gerariam um estado de energia positiva [efeito gravitacional positivo (repulsivo), equivalente à constante cosmológica $(\Lambda)$ ] capaz de compensar a atração gravitacional negati-

\footnotetext{
191 Physics Letters B108, p. 398, 1982.

192 Physical Review Letters, v. 48, p. 1220, 1982.

193 Proceedings of the 16th International Conference on High Energy Physics, 1972.

194 Physical Review Letters, v. 32, p. 438, 1974.

195 Physical Review D26, p. 2681, 1982.

196 Physics Letters B129, p. 177, 1983.
} 
va, de modo que a energia total do Universo seja completamente nula. É oportuno registrar que Linde, em 1974197, já havia apresentado a ideia de que um mecanismo similar ao Mecanismo de Higgs $(\mathrm{MH})$ poderia produzir o efeito gravitacional positivo referido acima. Note-se que este mecanismo (fundamental para explicar as massas das partículas vistas no histórico da MPPE) foi conceituado, em 1964, em trabalhos independentes, dos físicos, o inglês Peter Ware Higgs (n.1929; PNF, 2013) ${ }^{198}$, os belgas François Englert (n.1932; PNF, 2013) e Robert Brout (n.1928) ${ }^{199}$, e G. S. Guralnik, C. R. Hagen e o físico indiano Thomas Walter Bannerman Kibble (1932-2016) ${ }^{200}$.

\section{Radiação Cósmica de Fundo de Micro-onda}

Vistos os históricos dos MPPE e MPBB, vamos concluir este artigo sobre a RCFM. Conforme já registramos, antes do $\mathrm{BB}$, o nosso Universo era um estado de vácuo quântico (EVQ) com densidade de energia infinitamente grande e temperatura altíssima $\left(\approx 10^{32} \mathrm{~K}\right)$. Então, no instante do BB ( $\mathrm{t}=0 \mathrm{~s})$, houve criação de matéria (partícula) e de antimatéria (antipartícula) em quantidades iguais, assim como as quatro (4) forças da natureza (fg, fem, fr, fo) também foram "criadas", mas estavam acopladas, "supostamente" regidas por uma única (!?) lei física, envolvendo certas simetrias, sendo estas reunidas no hoje famoso Teorema $C P T$. Este, como já registramos, é baseado no princípio segundo o qual os sistemas físicos são invariantes para as transformações (invariância "gauge") que envolvem, concomitantemente, as operações de inversão da carga (C: troca de e por - e); inversão de paridade (P: troca de $\mathrm{r}$ por $-\mathrm{r})$; e inversão temporal $(\mathrm{T}$ : troca de $\mathrm{t}$ por $-\mathrm{t}$ ).

Quando o Universo começou a se esfriar, as 4 forças foram se desacoplando, o mesmo acontecendo com as simetrias e, em decorrência, foram surgindo novas leis físicas (hoje reunidas nos MPPE e MPBB). Com efeito, no final do tempo de Planck $\left(10^{-43} \mathrm{~s}\right)$, a fg desacoplou-se das outras três forças da Natureza e, em seguida, o Universo sofreu um período de expansão muito acelerada $\left[\left(10^{-35}-10^{-33}\right) \mathrm{s}\right]$, isto é, uma era inflacionária, tendo o Universo, em seu final, atingido uma temperatura de $\approx 10^{27} \mathrm{~K}$, ocasião em que a densidade de energia do EVQ transformou-se em "fótons muito quentes" (início da RCFM!?) em virtude do aniquilamento dos pares de partículas e antipartículas que se foram formando, segundo a Mecânica Quântica Relativística, tendo como base a Equação de Dirac (1928) (MQR/ED).

Decorrido $10^{-5} \mathrm{~s}$ do $\mathrm{BB}$, em que a temperatura do Universo era de $\approx 10^{13} \mathrm{~K}$, houve a quebra espontânea da $\mathrm{CP}$ (por um processo até hoje desconhecido) e, então, ocorreu a formação de 1 bilhão $\left(10^{9}\right)$ e 1 partículas contra 1 bilhão de antipartículas. Neste caso, no entanto,

\footnotetext{
197 Pis'ma v Zhurnal Eksperimental'́noi I Teoreticheskoi Fiziki, v. 19, p. 183, 1974.

198 Physics Letters, v. 12, 132; Physical Review Letters, v. 13, p. 508, 1964.

199 Physical Review Letters, v. 13, p. 321, 1964.

200 Physical Review Letters, v. 13, p. 585, 1964.
} 
devido também ao aniquilamento também estudado pelo $\mathrm{MQR} / \mathrm{ED}$, cada partícula restante é acompanhada de $10^{9}$ de fótons menos quentes (continuando a compor a RCFM!?) dos que os do final da era inflacionária.

Nesta oportunidade é fundamental acrescentar que, quando decorreram 380.000 anos, o Universo atingiu $\mathrm{T}=3.000 \mathrm{~K}$, ocasião em que se formou o primeiro átomo de $\mathrm{H}$, tornando o Universo transparente e permitindo que a RCFM chegasse até nós (en.wikipedia.org). É ainda oportuno dizer que, naquela época, as fem e fr se desacloparam da fo e constituíram a força eletrofraca que, como vimos antes, é composta pelo fóton $(\gamma)$, mediadora da fem, e pelo $Z^{0}$, um dos três mediadores da fr. Desse modo, foi possível existir o $\mathrm{H}$, pois o $\gamma$ faz o elétron girar em torno do próton, enquanto o $\mathrm{Z}^{0} \mathrm{o}$ faz girar em uma hélice de rosca-direita, que quebra a simetria (invariância) $P$.

Do que tratamos até aqui, surgem duas questões:

1) Por que existe uma assimetria matéria-antimatéria?

2) Que tipo são as partículas/antipartículas?

Uma das primeiras respostas a essas questões, foi apresentada pelo físico russo Andrey Dmitriyevich Sakharov (1921-1989; PNPaz, 1975), em 1967201, ao afirmar que assimetria devia-se à Quebra Espontânea da Simetria CP (QES-CP) (observada em 1964, como já anotamos), sobrando muito mais bárions [p. ex.: prótons (d.1919) e nêutrons (d, 1932)] do que antibárions [p. ex.: antiprótons (d.1955) e antinêutrons (d. 1956)]. Portanto, ele propôs o que hoje se conhece como processo de bariogênese, que é a base da nucleossíntese (Tabela Periódica dos Elementos), proposta por Alpher, Bethe e Gamow, em 1948, como já observamos antes.

Outra possível resposta para qual o tipo de partículas/antipartículas derivada da QES-CP, seriam os quarks/antiquarks (1964). Contudo, nesse caso, haveria uma nova questão. Com efeito, segundo já foi destacado, algumas das partículas hadrônicas são constituídas de três quarks iguais [p. ex.: $\Delta^{++}$(uuu) e $\Delta^{-}$(ddd) (d. 1953) e $\Omega^{-}$(sss) (d.1964)] e por serem os quarks partículas fermiônicas, essas três partículas violariam o PEP e, por isso, foi postulado que o quark possuiria mais um número quântico: a cor (1964). Por outro lado, segundo traduzido por fótons quentes), também poderia ser a origem da RCFM. É interessante registrar que em 1929202, os norte-americanos, o físico e químico Irving Langmuir (1881-1957; PNQ, 1932) e o físico Lewi Tonks (1879-1941), introduziram o conceito de plasma para representar um gás formado por partículas carregadas como, p. ex.: íons, que são átomos que perdem ou recebem elétrons e, sendo móvel, pelo menos um dos tipos de íons. No caso que estamos considerando, os “íons" são: bárions/antibárions ou quarks/antiquarks.

\footnotetext{
${ }^{201}$ Pis'ma Zhurnal Eksperimental'noi I Teoreticheskoi Fiziki, v. 5, p. 32; JETP Letters, v. 5, p. 24, 1967. 202 Physical Review, v. 33, p. 195, 1929.
} 
Para dar um fecho neste artigo, faremos um breve comentário sobre os dois processos de formação de partículas/antipartículas que originaram a RCFM. No bariogênese ${ }^{203}$, ele decorre de bárions [p. ex.: prótons (uud) e nêutrons (udd)] e de antibárions [por exemplo: antiprótons $(\bar{u} \bar{u} \bar{d})$ e antinêutrons a QCD (1973), os quarks/antiquarks (q/ $\bar{q})$ são ligados por uma força forte (fo) cujos mediadores são os glúons ( $\mathrm{g} / \bar{g}$ ), e não podem ser isolados. Em vista do exposto acima, após $10^{-5} \mathrm{~s}$ do $\mathrm{BB}$, aconteceu a formação de um plasma de quarks(antiquarks)-glúons(antiglúons), e o seu aniquilamento, $(\bar{u} \bar{d} \bar{d})]$ e no plasma de quarks(antiquarks)-glúons(antiglúons) (en.wikipedia.org), envolvem quarks/antiquarks (q/ $\bar{q})$. Ora, como nos dois casos, há participação inicial destes últimos (q/q ) e considerando que existem seis tipos de quarks ( $\mathrm{u}, \mathrm{d}, \mathrm{s}, \mathrm{c}, \mathrm{b}, \mathrm{t}$ ) e seus respectivos antiquarks $(\bar{u}, \bar{d}, \bar{s}, \bar{b}, \bar{t}$ ), surge então a pergunta: como o Universo, por ocasião de seu BG e que deu origem a sua formação atual, fez a escolha do tipo de quark? Por fim, ainda com relação à RCFM, cremos ser pertinente formular outra pergunta: por que os léptons [p. ex.: elétrons $\left(\mathrm{e}^{-}\right)$e pósitrons $\left(\mathrm{e}^{+}\right)$] que também contribuíram para aquela radiação (pois $\mathrm{e}^{-}+\mathrm{e}^{+} \rightarrow 2 \gamma$ ), não têm estrutura quarkônica?

Esperamos que o leitor possa acrescentar outras intrigantes questões.

203 en.wikipedia.org 